\title{
Mesenchymal Stem Cell-Derived Exosomes and Other Extracellular Vesicles as New Remedies in the Therapy of Inflammatory Diseases
}

\author{
Carl Randall Harrell ${ }^{1}$, Nemanja Jovicic ${ }^{2}{ }^{\oplus}$, Valentin Djonov ${ }^{3}$, Nebojsa Arsenijevic ${ }^{2}$ and \\ Vladislav Volarevic ${ }^{2, *}$ \\ 1 Regenerative Processing Plant, LLC, 34176 US Highway 19 N Palm Harbor, Palm Harbor, FL 34684, USA; \\ dr.harrell@regenerativeplant.org \\ 2 Department for Microbiology and Immunology, Center for Molecular Medicine and Stem Cell Research, \\ Faculty of Medical Sciences, University of Kragujevac, 69 Svetozar Markovic Street, \\ 34000 Kragujevac, Serbia; nemanjajovicic.kg@gmail.com (N.J.); arne@medf.kg.ac.rs (N.A.) \\ 3 Institute of Anatomy, University of Bern, 2 Baltzerstrasse, 3012 Bern, Switzerland; \\ valentin.djonov@ana.unibe.ch \\ * Correspondence: drvolarevic@yahoo.com; Tel./Fax: +381-3430-6800
}

Received: 30 October 2019; Accepted: 5 December 2019; Published: 11 December 2019

\begin{abstract}
There is growing evidence that mesenchymal stem cell (MSC)-based immunosuppression was mainly attributed to the effects of MSC-derived extracellular vesicles (MSC-EVs). MSC-EVs are enriched with MSC-sourced bioactive molecules (messenger RNA (mRNA), microRNAs (miRNAs), cytokines, chemokines, immunomodulatory factors) that regulate phenotype, function and homing of immune cells. In this review article we emphasized current knowledge regarding molecular mechanisms responsible for the therapeutic effects of MSC-EVs in attenuation of autoimmune and inflammatory diseases. We described the disease-specific cellular targets of MSC-EVs and defined MSC-sourced molecules, which were responsible for MSC-EV-based immunosuppression. Results obtained in a large number of experimental studies revealed that both local and systemic administration of MSC-EVs efficiently suppressed detrimental immune response in inflamed tissues and promoted survival and regeneration of injured parenchymal cells. MSC-EVs-based anti-inflammatory effects were relied on the delivery of immunoregulatory miRNAs and immunomodulatory proteins in inflammatory immune cells (M1 macrophages, dendritic cells (DCs), CD4+Th1 and Th17 cells), enabling their phenotypic conversion into immunosuppressive M2 macrophages, tolerogenic DCs and $\mathrm{T}$ regulatory cells. Additionally, through the delivery of mRNAs and miRNAs, MSC-EVs activated autophagy and/or inhibited apoptosis, necrosis and oxidative stress in injured hepatocytes, neurons, retinal cells, lung, gut and renal epithelial cells, promoting their survival and regeneration.
\end{abstract}

Keywords: mesenchymal stem cells; extracellular vesicles; therapy; immunosuppression; regeneration

\section{Introduction}

Epidemiological studies revealed a significant increase in the incidence of autoimmune and inflammatory diseases during the last two decades [1]. Accordingly, the total number of patients taking immunosuppressive drugs has been continuously increasing [2]. Long-term administration of immunosuppressive medications is inevitably associated with increased risk of infection and malignancy due to the sustained suppression of anti-microbial and anti-tumor immunity [3]. Therefore, new therapeutic agents, which could suppress detrimental immune response without causing life-threatening immunosuppression are urgently needed for the treatment of autoimmune and inflammatory diseases. 
Due to their capacity to modulate phenotype and function of immune cells, mesenchymal stem cells (MSCs) have been considered as potentially new remedy for the treatment of autoimmune and inflammatory diseases [4]. Although the large number of experimental and clinical studies demonstrated beneficial effects of MSCs in alleviation of immune cell-driven, organ-specific and systemic inflammatory disorders [4], several safety concerns related to the MSC-based therapy has been raised [5]. Unwanted differentiation of transplanted MSCs and their possible malignant transformation have been identified as the most important safety issues [5]. Additionally, patients suffering from inflammatory bowel diseases (IBDs) and idiopathic pulmonary fibrosis (IPF) who received immunosuppressive drugs just before MSC injection developed severe respiratory and gastrointestinal infections, indicating that MSCs should not be used immediately after or in combination with other immunosuppressive agents [6-8]. Autologous transplantation of MSCs is difficult to attempt on patients with fulminant diseases because of a long cell preparatory period and cell transplantation timing. Therefore, allogeneic MSCs have been used for the attenuation of acute and fulminant inflammation [4]. However, MSCs express major histocompatibility complex (MHC) class I molecules and may elicit strong allogeneic immune responses in MHC-class I-mismatched recipients that could result in life-threatening aggravation of on-going inflammation [9].

A large number of experimental and clinical studies revealed that most of MSC-based immunomodulatory effects were attributed to the immunoregulatory properties of MSC-sourced secretome, which consists of a soluble component and encapsulated extracellular vesicles (MSC-EVs): apoptotic bodies, microvesicles and exosomes (MSC-Exos) [10]. Subcellular particles derived from dead or dying MSCs might contribute to the therapeutic efficacy of transplanted MSCs [11]. Accordingly, during the apoptotic loss of MSCs, MSC-derived immunoregulatory factors are within large EVs (apoptotic bodies with diameter $>1000 \mathrm{~nm}$ ) delivered to the phagocytes, inducing alteration in their phenotype and function [11-13]. Microvesicles (100-1000 nm) and Exos (30-200 nm) are nano-sized MSC-sourced EVs, which are, after the budding from the plasma membrane, released into the extracellular milieu where it exerts biological effects in a paracrine and endocrine manner [14].

MSC-derived EVs express several adhesion molecules (CD29, CD44 and CD73), which enable their homing to the injured and inflamed tissues. In the mouse model of acute kidney injury (AKI), MSC-EVs were mainly accumulated in the inflamed kidneys [15], while in an intracerebral hemorrhage model, MSC-EVs were detected in the injured brains [12]. Nevertheless, most of intravenously injected MSC-EVs accumulate in the liver, spleen and the lungs where the mononuclear phagocyte system (MPS) is active [12]. Clearance of EVs from the circulation was much slower in macrophage-depleted mice, indicating the important role of MPS in EVs biodistribution. Therefore, several research groups used a membrane-editing technology to induce surface modifications of MSC-EVs in order to increase their chances for reaching the target cells before being taken up by the MPS. Alvarez and colleagues increased neurotropism of EVs by modifying their membrane protein Lamp2B with Rabies viral glycoprotein (RVG), which specifically binds to the acetylcholine receptors on neuronal cells. Accordingly, EVs displaying the RVG protein more specifically targeted neuronal cells and showed better therapeutic effects in attenuation of Alzheimer's disease than naïve EVs [16]. Kooijman and coworkers used glycosylphosphatidylinositol (GPI), a glycolipid, which is integrated into the EV's membrane during their biogenesis, to incorporate chemokine receptors, enzymes, antibodies and signaling molecules on EV's membranes, enhancing their tropism and therapeutic potential [17].

MSC-EV's membrane is enriched in cholesterol, sphingomyelin, ceramide and lipid raft proteins [9] enabling membrane fusion with target cells and trafficking of MSC-EVs through the body, regardless of biological barriers [10]. It was suggested that MSC-EVs, in a similar manner as tumor-derived EVs, crossed the blood-brain barrier (BBB) and that transcytosis is the main underlying mechanism [18]. Endothelial recycling endocytic pathway is involved in the transcellular transport of EVs [19]. Transcytosis through the brain endothelial cells includes clathrin-dependent and caveolin-independent endocytosis of EVs, their intracellular trafficking (driven by Rab11-expressing recycling endosomes) 
and vesicle associated membrane protein (VAMP)-3/Snap23/syntaxin 4-dependent release of EVs into the extracellular environment [20].

Upon reaching their target cells, MSC-EVs may trigger signaling via the receptor-ligand interaction, or be internalized by endocytosis to deliver their content [21]. All MSC-EVs are enriched with MSC-sourced bioactive molecules (messenger RNA (mRNA) and microRNAs (miRNAs)), enzymes, cytokines, chemokines, immunomodulatory and growth factors) that regulate phenotype, function, survival and homing of immune cells [14]. Accordingly, immunosuppressive effects elicited by MSC-EVs were similar to those observed after transplantation of MSCs [10]. As a cell-free product, MSC-derived EVs overcomes all safety concerns related to the long-term survival of engrafted MSCs, including their un-controlled differentiation, malignant alteration or rejection due to the activation of allogeneic immune response in MHC-mismatched recipients [14]. Importantly, composition of MSC-EVs can be modulated by MSCs' preconditioning in vitro, enabling generation of disease-specific, MSC-based, immunosuppressive product, which could be used as a new remedy in cell-free treatment of autoimmune and inflammatory diseases [14].

In this review article we emphasized current knowledge regarding molecular and cellular mechanisms responsible for the therapeutic effects of MSC-EVs in attenuation of autoimmune and inflammatory diseases. We described the disease-specific cellular targets of MSC-EVs and defined MSC-sourced molecules, which were mainly responsible for MSC-EV-based protection of injured cells and/or immunosuppression. An extensive literature review was carried out in October 2019 across several databases (MEDLINE, EMBASE, Google Scholar, ClinicalTrials.gov), from 1991 to present. Keywords used in the selection were: "mesenchymal stem cells (MSCs)", "extracellular vesicles (EVs)", "exosomes (Exos)", "inflammation", "macrophages", "dendritic cells", "neutrophils", “T cells", "gut epithelial cells", "hepatocytes", "hepatic stellate cells", "lung epithelial cells", "renal tubular cells", "retinal cells", "neurons" and "cardiomyocytes". All journals were considered, and an initial search retrieved 1837 articles. The abstracts of all these articles were subsequently reviewed by three of the authors (CRH, VD and VV) to check their relevance to the subject of this manuscript. Eligible studies had to delineate molecular and cellular mechanisms involved in the beneficial effects of MSC-derived EVs and their findings were analyzed in this review.

\section{Macrophages: The Main Cellular Targets of MSC-Derived EVs in Alleviation of Colon Inflammation}

Macrophages have been identified as the most important cells for the induction of colon inflammation [22,23]. Massive release of damage-associated molecular patterns (DAMPs) from injured epithelial cells activates NF- $\mathrm{KB}$ signaling pathway in colon macrophages, resulting in increased expression of inducible nitric oxide synthase (iNOS) and enhanced secretion of inflammatory cytokines (tumor necrosis factor alpha (TNF- $\alpha$ ), IL-1 $\beta$ ), nitric oxide (NO) and lymphocyte and monocyte-recruiting chemokines (CCL-17 and CCL-24) [24]. Macrophage-derived TNF- $\alpha$ and IL-1 $\beta$ induce enhanced expression of $\mathrm{E}$ and $\mathrm{P}$ selectins on endothelial cells enabling massive influx of circulating monocytes and lymphocytes in the injured gut [22]. Macrophage-sourced CCL-17 and CCL-24 attract inflammatory M1 macrophages and IFN $-\gamma$ producing CD4+Th1 cells, which either directly damage epithelial cells (NO-producing M1 macrophages) or activate macrophages in IFN- $\gamma$-dependent manner (Th1 cells) and indirectly promote colon injury and inflammation by enabling creation of "positive inflammatory loop" in the gut [22].

Several recently published studies indicated that MSC-based alleviation of colitis was mainly relied on MSC-EV-induced suppression of colon macrophages [25-28] (Figure 1). Cao and colleagues showed that MSC-EVs significantly alleviated dextran sulphate sodium (DSS)-induced colitis in mice by inducing polarization of colon macrophages in immunosuppressive, M2 phenotype [25]. Higher number of IL-10-producing M2 macrophages, observed in MSC-EVs-treated mice, correlated with reduced weight loss, alleviated injury of gut epithelial cells and increased colon length [25]. Concentration of macrophage-sourced inflammatory cytokines and chemokines (TNF- $\alpha$, CCL-17 and CCL-24) and 
Th1-derived IFN- $\gamma$ were significantly attenuated in the gut of DSS-treated mice that received MSC-EVs. Additionally, MSC-EVs managed to increase colon concentration of immunosuppressive cytokines (IL-10 and transforming growth factor beta (TGF- $\beta$ )), enabling enhanced repair and regeneration of DSS-injured epithelial cells [25]. Importantly, in vitro obtained results confirmed that MSC-EVs entered in lipopolysaccharides (LPS)-activated colon macrophages, suppressed production of inflammatory cytokines and induced generation of immunosuppressive M2 phenotype [25].
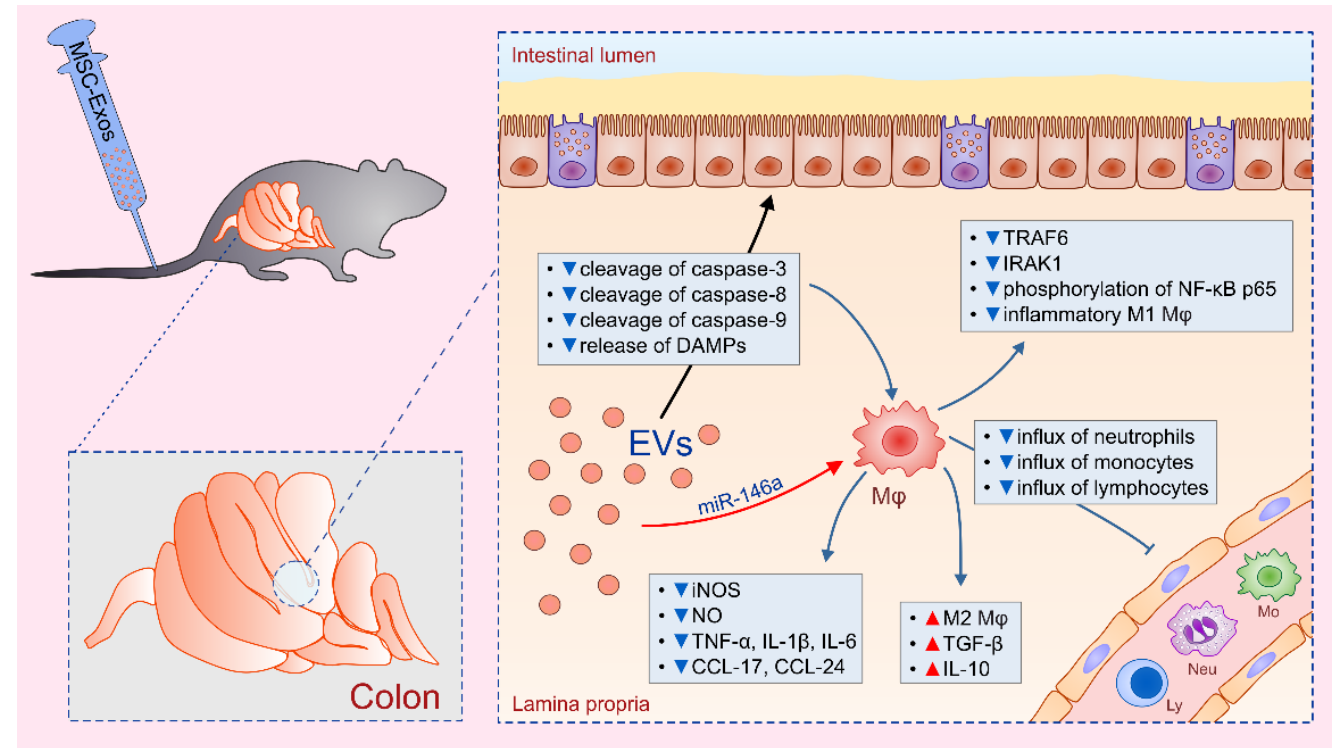

Figure 1. Modulation of phenotype and function of colonic macrophages as the main mechanism for mesenchymal stem cell-derived extracellular vesicle (MSC-EV)-based attenuation of ulcerative colitis: MSC-EVs reduced cleavage of caspase-3, -8 and -9 and alleviated release of damage-associated molecular patterns (DAMPs) from injured gut epithelial cells, resulting in attenuated activation of NF- $\kappa B$ signaling pathway in colon macrophages. Through the delivery of miR-146a, MSC-EVs inhibited TNF receptor-associated factor 6 (TRAF6) and IL-1 receptor-associated kinase 1 (IRAK1) expression, down-regulated phosphorylation of NF- $\mathrm{B}$ p 65 and inhibited generation of inflammatory M1 phenotype in macrophages, which was manifested by down-regulated expression of inducible nitric oxide synthase (iNOS), significantly reduced production of nitric oxide (NO), inflammatory cytokines (TNF- $\alpha$, IL-1 $\beta$, IL-6) and chemokines (CCL-17 and CCL-24) and resulted in reduced influx of circulating neutrophils, monocytes and lymphocytes in the inflamed gut. Additionally, MSC-EVs induced polarization of colon macrophages in anti-inflammatory M2 phenotype, manifested by increased secretion of immunosuppressive cytokines TGF- $\beta$ and IL-10 and alleviation of colitis.

The main mechanism responsible for MSC-EV-induced inhibition of colon macrophages relies on the suppression of NF- $\mathrm{KB}$ and iNOS-driven signaling [26,27]. Administration of MSCs-EVs down-regulated expression of NF- $\mathrm{KB}$ p65 and reduced production of NO, IL- $1 \beta$ and IL-18 in colon macrophages, resulting in alleviated 2,4,6-trinitrobenzene sulfonic acid (TNBS)-induced colitis [26,27]. $\mathrm{Wu}$ and coworkers suggested that microRNA-146a (miR-146a), a well-known anti-inflammatory miRNA, acted as a negative feedback regulator of colon macrophages in MSC-EV-based alleviation of gut inflammation [27]. Administration of EVs, obtained from miR-146-overexpressing MSCs, inhibited TNF receptor-associated factor 6 (TRAF6) and IL-1 receptor-associated kinase 1 (IRAK1) expression, down-regulated phosphorylation of NF- $\mathrm{kB}$ p 65 and inhibited generation of inflammatory phenotype in macrophages, attenuated production of TNF- $\alpha$, IL-1 $\beta$, IL- 6 and reduced colon injury and inflammation [27].

Yang and colleagues suggested that modulation of anti-oxidant/oxidant balance in the injured gut was responsible for MSC-EVs-induced effects on macrophage phenotype and function [26]. MSC-EV-mediated suppression of NO-driven injury in the gut was accompanied by decreased activity 
of myeloperoxidase and malondialdehyde and increased superoxide dismutase and glutathione activity. Furthermore, MSC-EVs reduced cleavage of caspase-3, -8 and -9 and alleviated release of DAMPs from injured gut epithelial cells, resulting in attenuated activation of NF- $\mathrm{kB}$ signaling pathway in colon macrophages, which consequently led to the generation of immunosuppressive M2 phenotype [26].

Mao and coworkers suggested that, in addition to the inhibition of NF-KB and iNOS, MSC-EVs exert their beneficial effects in colitis through the inhibition of IL-7 signaling in colon macrophages, as well [28]. IL-7 displays strong chemotactic property for circulating monocytes enabling their massive accumulation in inflamed tissues [29]. Additionally, IL-7 induces increased production of NO, TNF- $\alpha$ and IL-1 $\beta$ in macrophages, enhancing their inflammatory properties [29]. MSC-EVs contain miR17, which impairs IL-7:IL-7 receptor signaling by preventing synthesis and transactivation of Janus kinase $1[30,31]$. In line with these findings, MSC-EVs treatment significantly reduced activation of IL-7 and iNOS-signaling pathways in colon macrophages, resulting in attenuated production of TNF- $\alpha$, IL-1 $\beta$, IL-6 and increased secretion of IL-10, which led to the alleviation of colitis [28].

\section{Molecular Mechanisms Responsible for MSC-EVs-Based Protection of Hepatocytes in Acute Liver Injury and Fibrosis}

As recently evidenced by us and others, MSC-derived secretome efficiently attenuated acute liver failure and liver fibrosis in mice by suppressing major effector cells: natural killer T cells (NKT) cells in fulminant hepatitis and CD4+ Thelper lymphocytes and hepatic stellate cells (HSCs) in fibrosis [32-36].

MSC-sourced secretome contains high concentration of $\mathrm{NO}$ and reactive nitrogen species, which decrease proliferation of liver NKT cells [37]. Accordingly, administration of MSC-derived secretome significantly reduced total number of inflammatory NKT cells in the injured livers of mice with fulminant hepatitis [32,34]. Additionally, MSC-derived secretome contains Kynurenine, which maintains immunosuppressive phenotype of FoxP3-expressing NKT cells in the inflamed livers and suppress their transdifferentiation in inflammatory, IL-17-producing NKT17 cells [34]. Liver NKT cells cultured in the presence of MSC-sourced secretome have reduced capacity for production of hepatotoxic (TNF- $\alpha$ ) and inflammatory cytokines (IFN- $\gamma$, IL-17) [32,34]. Moreover, reduced expression of molecules, which are responsible for NKT cell-dependent apoptosis of hepatocytes (Fas ligand, CD107a and NKG2D) was observed in liver NKT cells cultured in the presence of MSC-derived secretome [34].

In addition to immunosuppressive effects against NKT cells, MSC-sourced secretome may directly protect hepatocytes from cell death [38,39]. Injection of human menstrual blood-derived MSC-Exos significantly attenuated D-galactosamine/lipopolysaccharide (D-GalN/LPS)-induced acute liver injury and increased survival rate of experimental mice by suppressing caspase-3-driven apoptosis of hepatocytes [38]. In line with these findings are results obtained by Chen and colleagues who provided additional evidence of anti-apoptotic capacity of MSC-EVs in a murine model of autoimmune hepatitis (AIH) [39]. Hepatoprotective effects of MSC-Exos were relied on suppression of NLRP3-dependent activation of caspase- 1 and on inhibition of caspase-1-driven pyroptosis, characterized by plasma membrane rupture, cytoplasmic swelling, osmotic lysis, DNA cleavage and massive release of pro-inflammatory cytokines (IL-1 $\beta$ and IL-18) [40]. Accordingly, by suppressing pyroptosis, MSC-Exos inhibited cell death of hepatocytes and attenuated IL-1 $\beta$ and IL-18-driven inflammation. MSC-derived miR-233 was crucially important for these hepatoprotective effects of MSC-Exos since administration of Exos derived from miR-233 deficient MSCs did not attenuate AIH [39]. The analysis of NLRP3-signaling pathway revealed that exosomal miR-233 suppressed NLRP3:caspase-1-induced pyroptosis by inducing degradation of NLRP3 mRNA in hepatocytes [39]. MSC-Exos attenuate oxidative stress in inflamed livers, as well [41]. MSC-Exo-derived glutathione peroxidase 1 (GPX1) was mainly responsible for MSC-Exo-dependent suppression of reactive oxygen species (ROS) formation in injured hepatocytes [41].

In addition to their hepatoprotective effects, MSC-Exos may induce proliferation of hepatocytes. As recently evidenced by Du and colleagues intravenous injection of Exos, obtained from human-induced 
pluripotent stem cell-derived MSCs (hiPSC-MSCs-Exos) attenuated hepatic ischemia-reperfusion (I/R) injury by suppressing necrosis of hepatocytes and by promoting their proliferation [42]. The serum levels of hepatocyte injury markers (aspartate aminotransferase (AST) and alanine aminotransferase (ALT) were significantly lower and the expression levels of proliferation markers (proliferation cell nuclear antigen (PCNA) and phosphohistone-H3 (PHH3)) were greatly increased in the livers of I/R-injured mice that received hiPSC-MSCs-Exos [42]. Significantly increased proliferation of hiPSC-MSCs-Exos-treated primary hepatocytes and HL7702 human hepatocytes was confirmed in vitro. Mechanistically, hiPSC-MSCs-Exos directly fused with target hepatocytes or HL7702 cells and increased the activity of sphingosine kinase (SK1) resulting in synthesis of sphingosine-1-phosphate (S1P), which promoted hepatocyte growth, survival and proliferation [42,43]. This phenomenon was completely abrogated after inhibition of either SK1 or S1P receptor, confirming crucial importance of SK1/S1P signaling for hiPSC-MSCs-Exos-induced enhanced proliferation of hepatocytes [42].

Several lines of evidence demonstrated that MSC-EVs protected hepatocytes during chronic liver inflammation and fibrosis, as well [44]. Results obtained by Li and colleagues showed that human umbilical cord-MSCs-derived Exos attenuated carbon tetrachloride (CCl4)-induced liver fibrosis in mice, as evidenced by recovered serum AST levels and reduced deposition of collagen type I and III in the liver [44]. Significantly decreased expression of TGF- $\beta 1$ and phosphorylated Smad 2 was observed in the CCl4-injured livers of MSC-Exo-treated mice, indicated that MSC-Exo-dependent inhibition of TGF- $\beta 1$ signaling pathway in hepatocytes was crucially important for anti-fibrotic effects of MSC-Exos. Upon phosphorylation, Smad2 formed complexes with phosphorylated Smad3 and Smad4 and, subsequently, translocated into the nucleus to regulate the transcription of genes responsible for epithelial-to-mesenchymal transition (EMT) of hepatocytes [45]. Significant increase in E-cadherin-positive cells and decrease in N-cadherin- and vimentin-positive cells in MSC-Exo-treated fibrotic livers, suggested that MSC-Exos prevented TGF- $\beta 1 /$ Smad2-induced EMT of hepatocytes [44]. This hypothesis was confirmed in vitro. MSC-Exos completely reversed spindle-shaped morphology and abrogated expression of EMT-associated markers in HL7702 human hepatocytes that underwent EMT after treatment with recombinant TGF- $\beta 1$ [44].

MSC-EVs attenuated chronic liver inflammation by suppressing production of inflammatory cytokines (TNF- $\alpha$, IL-1 $\beta$ and IL-6) and pro-fibrotic TGF- $\beta 1$ in liver macrophages (Kupffer cells), while HSCs were the main cellular targets in MSC-EVs-based alleviation of liver fibrosis [46]. Through the production of inflammatory cytokines and monocyte and lymphocyte-attracting chemokines, Kupffer cells attract circulating leucocytes in inflamed liver contributing to the progression of inflammation [47]. Furthermore, through the production of TGF- $\beta 1$, Kupffer cells induce enhanced expression of pro-fibrotic genes (collagen I, vimentin, $\alpha$-SMA and fibronectin) in HSCs, resulting in the development of liver fibrosis [47]. In line with these findings, $\mathrm{Qu}$ and coworkers engineered miRNA-181-5p-overexpressing adipose tissue derived MSCs (MSCs ${ }^{\text {miRNA-181-5p }}$ ), which produced Exos that efficiently alleviated liver fibrosis by affecting survival and pro-fibrotic function of HSCs [48].

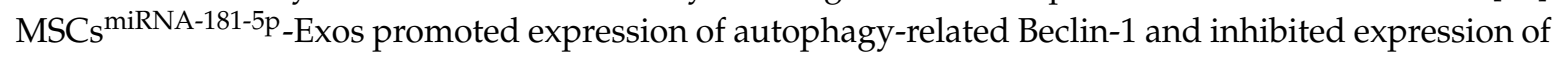
anti-apoptotic Bcl-2 in HSCs, resulting in increased apoptosis and autophagy of HSCs in fibrotic livers.

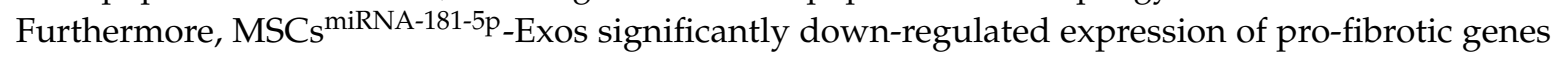
(collagen I, vimentin, $\alpha$-SMA and fibronectin) in HSCs, which led to the attenuation of CCl4-induced liver fibrosis in MSCs ${ }^{\text {miRNA-181-5p-Exos-treated mice [48]. }}$

\section{MSC-EVs as Next-Generation Therapeutics for the Treatment of Lung Inflammatory Diseases}

There is growing evidence that MSC-EVs protect lung epithelial cells from reactive oxidative species and proteolytic enzymes released by lung-infiltrating neutrophils and monocytes [49-52]. $\mathrm{Li}$ and colleagues demonstrated that MSC-EV-based protection of lung epithelial cells against oxidative stress-induced cell death is dependent on anti-apoptotic properties of miR-21-5p [50]. Intratracheal administration of MSC-Exos inhibited both intrinsic and extrinsic apoptotic pathways in lung epithelial cells. However, pre-treatment of MSCs with miR-21-5p antagomir completely abrogated 
MSC-Exos-mediated suppression of caspase-3, -8 and -9 and diminished MSC-Exo-based protective effects [50]. Western blot analysis revealed that pro-apoptotic phosphatase and tensin homolog (PTEN) and programmed cell death protein 4 (PDCD4) were the main targets of MSC-derived miR-21-5p since their expression was significantly decreased in lung epithelial cells of MSC-Exo-treated mice. When I/R-injured mice received Exos derived from miR-21-5p-antagomir-treated MSCs, expression of PTEC and PDCD4 and apoptosis of lung epithelial cells were not reduced, indicating crucial importance of miR-21-5p-dependent suppression of PTEN and PDCD4 for anti-apoptotic effects of MSC-Exos in I/R lung injury [50].

In addition to their anti-oxidative effects, MSC-EVs may protect lung epithelial cells by regulating protease/antiprotease balance in the inflamed lungs [51]. Alpha-1-antitrypsin (AAT) is a potent inhibitor of neutrophil-derived proteolytic enzymes, which protects lung epithelial cells and exerts important anti-inflammatory and immunomodulatory effects in the lungs [52]. Most recently, Bari and colleagues revealed that AAT was aggregated and/or adsorbed on the surface of adipose-tissue derived MSC-EVs that served as natural carriers of AAT, promoting its stability and activity in vivo [51]. Importantly, MSC-EVs derived from IL- $\beta$-primed MSCs showed significantly higher expression of AAT gene and had increased anti-elastase activity compared to MSC-EVs obtained from IL- $\beta$-non-primed MSCs [51].

Importantly, MSC-EVs, in addition to AAT, contained 46 proteins involved in the response to Gram-negative bacteria, implying potent anti-microbial activity of MSC-EVs [51]. In line with these findings are results obtained by Hao and colleagues who demonstrated that administration of MSC-EVs remarkably reduced severity of bacterial pneumonia in mice [53]. MSC-EVs increased phagocytic and anti-microbial activity of lung-infiltrating neutrophils and monocytes by promoting synthesis of leukotriene B4 (LTB4) [53]. LTB4 is well-known activator of leucocytes, which augments phagocytosis and promotes release of anti-microbial agents, contributing to the bacterial clearance [54]. Hao and colleagues demonstrated that miR-145, contained within MSC-EVs, reduced expression of multidrug resistance-associated protein 1 (MRP1) in lung macrophages [53]. MRP1 is ATP-binding cassette transporter, which inhibits synthesis and release of LTB4 [53]. Accordingly, MSC-EV-induced suppression of MRP1 resulted in enhanced release of LTB4 by alveolar macrophages that, due to its anti-microbial activity, increased bacterial clearance and reduced severity of bacterial pneumonia in mice [53].

It is important to highlight that capacity of MSC-EVs to modulate phenotype and function of alveolar macrophages depends on the phase of anti-microbial inflammatory response [55]. During the onset of inflammation, MSC-EVs, in a miR-145/LTB4-dependent manner, promote phagocytic activity of alveolar macrophages contributing to the elimination of bacterial pathogens from the lungs. However, during the resolution of inflammation, MSC-EVs promote expansion of alternatively activated M2 macrophages that are involved in tissue repair and regeneration [55]. It is well known that alveolar macrophages, through the production of inflammatory cytokines and chemokines, orchestrate influx of circulating monocytes and lymphocytes in inflamed lungs, promoting chronic inflammation [55]. Therefore, MSC-EV-based suppression of chronic, macrophage-driven inflammatory lung diseases was mainly relied on MSC-EV-dependent polarization of alveolar macrophages. MSC-Exos significantly decreased iNOS mRNA expression and remarkable increased expression of Arginase-1 mRNA in alveolar macrophages, inducing their polarization from inflammatory M1 towards immunosuppressive M2 phenotype [50,55]. Accordingly, concentration of M1-related inflammatory cytokines (IL-8, IL-1 $\beta$, IL- 6 and TNF- $\alpha$ ) was significantly reduced and concentration of M2 macrophage-derived immunosuppressive cytokines (IL-10 and TGF- $\beta$ ) was increased in the lungs of I/R-injured mice that received MSC-Exos [50].

Interestingly, as recently revealed by Huang and colleagues, aging MSC-EVs did not manage to induce generation of M2 macrophages in the inflamed lungs [56]. Although aging and young MSC-EVs had similar phenotypic characteristics (expression of CD63, CD81, CD105 and CD44), their capacity to alter the phenotype of alveolar macrophages was different. Internalization of aging MSC-EVs by alveolar macrophages was significantly lower compared to the young MSC-EVs. Furthermore, aging 
MSC-EVs had reduced capacity to inhibit production of inflammatory, M1-related cytokines (IL-6, IL-1 $\beta$ and TNF- $\alpha$ ) and to induce expression of M2-related Arginase-1 in alveolar macrophages [56]. Most importantly, aging and young MSC-EVs differed in levels of miRNAs (miR-223-5p, miR-127-3p and miR-125b-5p) that regulate macrophage polarization. Compared with aging MSC-EVs, young MSC-EVs showed higher expression of miR-223-5p (which is responsible for induction of M2 phenotype in alveolar macrophages) and lower expression of miR-127-3p and miR-125b-5p (which promote generation of M1 phenotype in macrophages) [56]. Since aging MSC-Exos had significantly reduced capacity to attenuate M1 macrophage driven inflammation in the lungs, MSC-Exos used for the therapy of inflammatory lung diseases should be obtained only from young donors.

Mansouri and colleagues recently revealed that single intravenous administration of Exos, obtained from human bone marrow-derived MSC, managed to significantly attenuate bleomycin-induced lung fibrosis in mice through the modulation of phenotype and function of alveolar macrophages [57]. An improved Ashcroft score and reduced deposition of collagen were observed in bleomycin-injured lungs of MSC-Exo-treated animals. MSC-Exo-based alleviation of fibrosis was followed by significantly reduced number of TGF- $\beta 1$-producing, Arginase-1 and CD206-expressing alveolar macrophages, indicating that macrophages were the main cellular targets of MSC-Exos in alleviation of pulmonary fibrosis. Importantly, anti-fibrotic effects were not observed in bleomycin-injured mice that received fibroblasts-derived Exos or Exos free iodixanol, suggesting that immunomodulatory properties of MSCs were responsible for beneficial effects of MSC-Exos [57].

In addition to alveolar macrophages, MSC-EVs may also modulate phenotype and function of lung-infiltrating dendritic cells (DCs) [58]. As recently evidenced by Cho and colleagues, MSC-EV-based alleviation of Th2 cell-driven immune response against Aspergillus protease antigen was dependent on suppression of antigen-presenting properties of DCs [45]. MSC-Exos induced increased expression of immunosuppressive IL-10 and TGF- $\beta$ that suppressed maturation of lung DCs [58]. Immature DCs of MSC-Exos-treated mice had reduced expression of co-stimulatory molecules (CD40, CD80 and CD86) and were not capable to optimally activate $\mathrm{CD} 4+\mathrm{Th} 2$ cells, resulting in alleviation of Th2 cell-driven lung inflammation [58].

The lung is a portal of entry for numerous microbial pathogens, which are, immediately after invasion, captured and efficiently eliminated by alveolar macrophages and lung DCs, resulting in the activation of antigen specific, $T$ cell-driven immune response $[59,60]$. Upon activation, alveolar macrophages and lung DCs produce large amount of inflammatory chemokines and cytokines and orchestrate both local and systemic immune response [59]. Accordingly, lung macrophages and DCs have been considered as the cells that are crucially important for the generation and development of chronic inflammatory diseases [59]. Since most of intratracheally and intravenously administered MSC-EVs accumulate in the lungs where, in similar manner as microbial pathogens, become phagocyted by lung-infiltrated macrophages and DCs, capacity of MSC-EVs to modulate phenotype and function of these professional antigen-presenting cells could be used not only for alleviation of inflammatory lung diseases but also for modulation of detrimental macrophage and DC-driven systemic immune response.

\section{Modulation of Microglial Activity: The Main Mechanism Responsible for MSC-EVs-Dependent Attenuation of Neuroinflammatory Diseases}

Microglia, the resident immune cells of the central nervous system (CNS), maintain tissue homeostasis under physiological conditions [61]. However, after neuronal injury, microglia secrete pro-inflammatory cytokines that either have direct neurotoxic effects or, in combination with inflammatory chemokines, promote influx of circulating neutrophils in inflamed tissue [61]. An excessive microglial activation damages the surrounding healthy neural tissue and induces the release of alarmins and DAMPs from dead or dying neurons, which in turn, activates microglia enabling creation of "positive inflammatory loop" in CNS, that results in a massive and progressive loss of neurons [61]. In line with these findings, Ding and colleagues recently revealed that modulation of microglial activity was the main mechanism responsible for beneficial effects of MSC-EVs in 
alleviation of Alzheimer's disease (AD) [62]. Excessive accumulation of the amyloid- $\beta$ peptide (A $\beta$ ) in the brain is considered as the most common pathological characteristic of $\mathrm{AD}$, which triggers dysfunction of cognitive behavior [63]. Intravenously injected Exos, obtained from human umbilical cord-derived MSCs, managed to reduce A $\beta$ deposition and increased spatial learning and memory function in A $\beta P P / P S 1$ transgenic mice, used as murine model of AD [62]. Additionally, Bodart-Santos and colleagues recently revealed that MSC-EVs prevented neuronal damage in AD by suppressing oxidative stress-induced injury of hippocampal neurons [64]. Catalase was mainly responsible for MSC-EV-based protection against ROS-induced injury since MSC-EVs with inactivated catalase were unable to prevent ROS formation in hippocampal neurons [64]. MSC-Exos induced polarization of microglia towards immunosuppressive M2 phenotype. Significantly higher number chitinase 3-like 3, arginase-1 and mannose receptor $C$ type 1 (MRC1)-expressing $\mathrm{M} 2$ microglia cells were found in the brains of MSC-Exos-treated A $\beta$ PP/PS1 mice [62]. M2 cells produce A $\beta$-degrading enzymes (neprilysin (NEP) and insulin-degrading enzyme (IDE)) and anti-inflammatory cytokines (IL-10 and TGF- $\beta$ ), contributing to the reduced $A \beta$ deposition and alleviated inflammation [61]. Significantly increased levels of NEP, IDE, IL-10 and TGF- $\beta$, and greatly reduced concentration of inflammatory cytokines (TNF- $\alpha$ and IL-1 $\beta$ ) were noticed in the brains of MSC-Exos-treated A $\beta$ PP/PS1 mice, indicating that MSC-Exos induce conversion of microglia from inflammatory M1 towards immunosuppressive M2 phenotype [62]. MSC-Exo-induced alternative microglial activation was confirmed in vitro, since significantly higher concentration of IL-10 and TGF- $\beta$ and lower concentration of TNF- $\alpha$ and IL-1 $\beta$ were measured in supernatants of MSC-Exo-treated BV2 murine microglia cells [62].

Modulation of microglial activity was mainly responsible for beneficial effects of MSC-Exos in alleviation of multiple sclerosis (MS), inflammation-mediated demyelinating disease [65]. Significantly improved motor function was noticed in Theiler's murine encephalomyelitis virus (TMEV)-infected mice that received MSC-EVs [65]. Remarkably reduced number of Iba-1-positive microglia cells was observed in the brains of TMEV+MSC-EV-treated mice compared to TMEV-only treated animals [65]. Importantly, MSC-EVs altered cytokine milieu in TMEV-infected mice. Significantly lower concentration of microglia-derived inflammatory cytokines (TNF- $\alpha$, IL-1- $\beta$, IL-18, IL-6 and IL-12) was noticed in TMEV+MSC-EV-treated mice [65]. Furthermore, MSC-EVs significantly alleviated concentration of Th1 cell-derived IFN- $\gamma$ and Th17 cell-sourced IL-17A, indicating that, in addition to microglia, MSC-EVs suppressed inflammatory properties of brain-infiltrating inflammatory CD4+T cells, as well [65].

As recently revealed by Shiue and colleagues [66], continuous intrathecal injection of MSC-Exos enabled functional recovery from nerve ligation-induced injury [66]. MSC-Exos suppressed production of inflammatory cytokines (TNF- $\alpha$ and IL-1 $\beta$ ) and promoted synthesis of anti-inflammatory cytokines (IL-10 and TGF- $\beta$ ) in microglia, resulting in the alleviation of inflammation within the site of neural injury [66]. The analgesic effects of MSC-Exos involved their actions on neurons, as well. MSC-Exos delivered brain-derived neurotrophic factor and glial cell line-derived neurotrophic factor in the ipsilateral L5/6 dorsal root ganglion of nerve-ligated rats, enabling better recovery from nerve ligation-induced injury [66]. Protein analysis demonstrated that vascular endothelial growth factor C, angiopoietin-2 and fibroblast growth factor- 2 were also present in the MSC-Exos, indicating that induction of neo-angiogenesis may be, at least partially responsible for beneficial effects of MSC-Exos. Importantly, immunofluorescence staining showed that MSC-Exos were presented in the ipsilateral L5 spinal dorsal horn, dorsal root ganglion and peripheral axons, suggesting a high homing ability of MSC-Exos [66].

Huang and colleagues provided evidence that MSC-Exos ameliorated cerebral I/R injury by preventing neural cell death through the inhibition of caspase-9 and caspase-3 [67]. MSC-sourced pigment epithelium-derived factor (PEDF), which exhibits anti-inflammatory, antioxidative and neuroprotective properties, was mainly responsible for beneficial effects of MSC-Exos [68]. Through the delivery of PEDF, MSC-Exos increased expression of autophagy-associated protein LC3 and suppressed caspase-3-driven apoptosis in neurons, significantly reducing I/R-induced injury [67]. Exos, obtained from PEDF-overexpressing MSCs showed better therapeutic effects, while inhibition of autophagy 
significantly reduced neuroprotection elicited by PEDF-containing MSC-Exos, indicating crucial importance of PEDF-induced autophagy for MSC-Exo-based attenuation of cerebral I/R injury [67].

\section{Molecular Mechanisms Responsible for MSC-EVs-Based Renal Protection}

MSC-EVs-dependent renal protection is relied on the inhibition of apoptosis, necrosis and oxidative stress in renal tubular epithelial cells as well as suppression of detrimental immune response in the kidneys (Figure 2) [69]. MSC-sourced mRNAs, miRNAs and immunosuppressive factors were mainly responsible for beneficial effects of MSC-EVs in alleviation of acute and chronic renal inflammation [69-80].

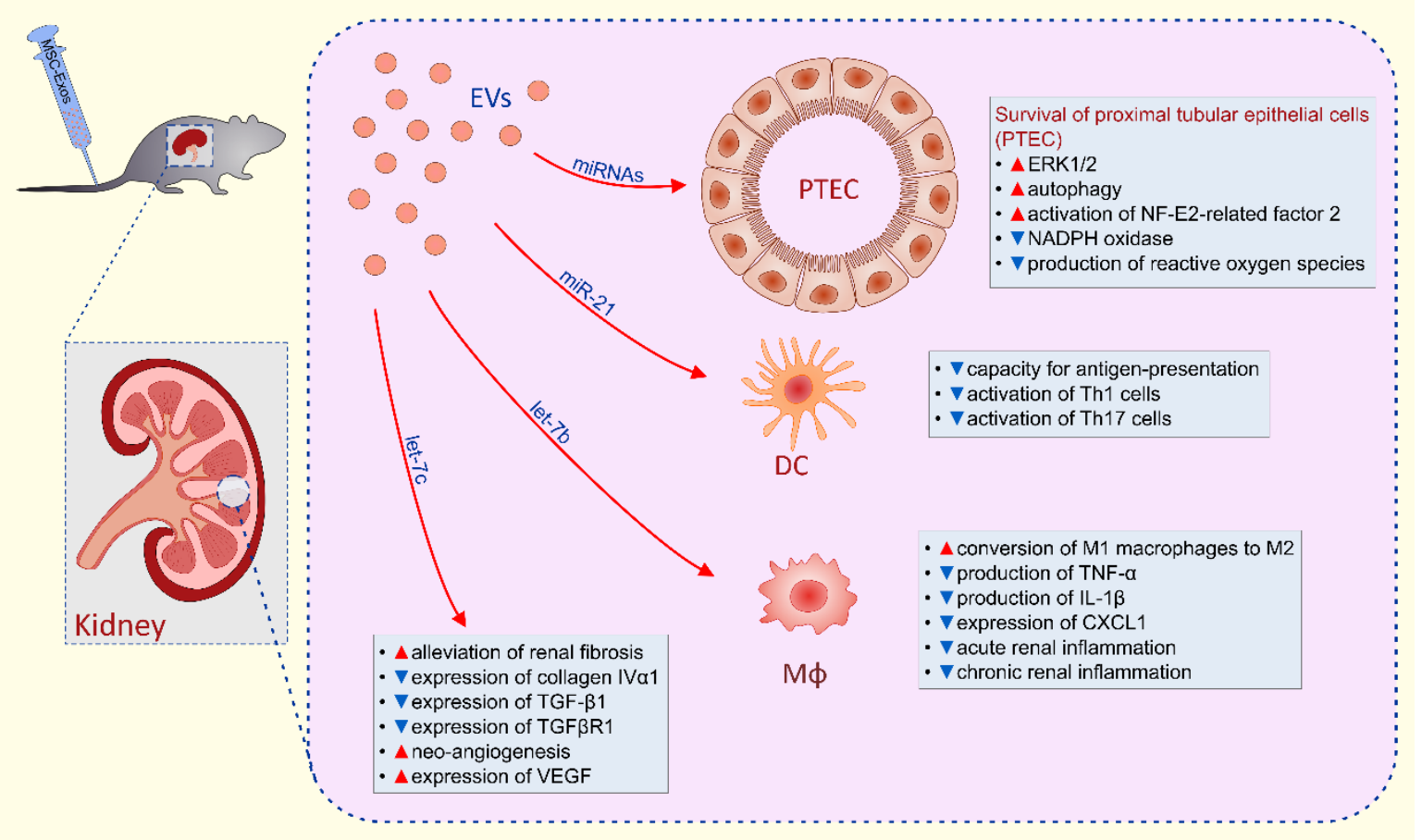

Figure 2. Molecular mechanisms responsible for MSC-EVs-based renal protection: MSC-EVs-dependent renal protection during acute kidney injury (AKI) is relied on inhibition of apoptosis, necrosis and oxidative stress and the promotion of autophagy in renal tubular epithelial cells as well as suppression of detrimental immune response. Through the delivery of messenger RNAs (mRNAs), MSC-EVs induce enhanced expression of ERK1/2 and promote survival of proximal tubular epithelial cells (PTEC). MSC-EVs activated autophagy in PTEC and protected against cisplatin-induced AKI by delivering trophic factor 14-3-3 , which interacted with ATG-16L, a protein essential for autophagy induction. MSC-EVs enhanced activation of NF-E2-related factor 2/antioxidant responsive element, decreased expression of NADPH oxidase and reduced production of reactive oxygen species in ischemic kidneys and promoted their regeneration. Additionally, through the delivery of miR-21, MSC-EVs significantly attenuated capacity for antigen-presentation of renal dendritic cells, which resulted in reduced activation of Th1 and Th17 cells and alleviation of Th1 and Th17 cell-driven inflammation in the kidneys. Through the delivery of microRNAs (miRNAs), particularly let-7b, MSC-EVs induced conversion of inflammatory M1 macrophages into immunosuppressive M2 cells, which produced lower amount of inflammatory cytokines (TNF- $\alpha$ and IL-1 $\beta$ ) and chemokine CXCL1, resulting in alleviated acute and chronic renal inflammation. MSC-sourced miRNA, particularly let-7c, targeted pro-fibrotic genes (collagen IV $\alpha 1$, TGF- $\beta 1$ and TGF $\beta$ R1) in inflamed kidneys, crucially contributing to the therapeutic effects of MSC-EVs in renal fibrosis. Additionally, neo-angiogenesis, induced by MSC-derived vascular endothelial growth factor (VEGF) was also responsible for beneficial effects of MSC-EVs in alleviation of renal fibrosis. 
Several lines of evidence demonstrated that MSC-derived mRNAs were involved in MSC-EVs-based attenuation of acute kidney injury (AKI) [70-73]. Bruno and colleagues noticed significantly improved renal function in glycerol and cisplatin-injured kidneys of experimental animals [70-72]. They revealed that mRNAs, which regulate transcription (e.g., CLOCK, IRF6 and LHX6), cell cycle regulation (e.g., SENP2, RBL1 and CDC14B) and DNA/RNA repair (e.g., HMGN4, TOPORS and ESF1) were contained within MSC-EVs and suggested that these MSC-derived mRNAs were mainly responsible for increased proliferation and suppressed apoptosis of renal tubular cells in cisplatin + MSC-EV-treated animals [70-72]. Pretreatment with ribonucleases (RNase) completely abolished MSC-EVs-based renoprotection, confirming that MSC-derived mRNAs were crucially involved in MSC-EV-dependent alleviation of AKI [70-72]. The same conclusion was made by Ju and coworkers who observed that RNase treatment abolished MSC-EVs-induced overexpression of ERK1/2 in renal tubular epithelial cells and completely abrogated therapeutic effects of MSC-EVs in I/R-induced AKI [73].

In line with these findings are results reported by Gatti and colleagues who demonstrated that MSC-EVs alleviated I/R-induced AKI by reducing apoptosis and by increasing proliferation of renal tubular cells [74]. Similarly as it was observed by Bruno et al. [70] and Ju and et al [73], MSC-EV-based renoprotection was diminished by RNase pretreatment [74], confirming the hypothesis that beneficial effects of MSCs-EVs were mainly mediated by MSC-sourced mRNA.

Wang and colleagues indicated that activation of autophagy in proximal tubular epithelial cells (PTEC) was responsible for greatly improved renal function of cisplatin + MSC-EVs-treated mice [75]. They showed that beneficial effects of MSC-EVs were completely abrogated by autophagy inhibitor, 3-methyladenine. Similarly, Jia and coworkers demonstrated that MSC-EVs activated autophagy in cisplatin-injured PTEC and protected against AKI by delivering trophic factor 14-3-3 , which interacted with ATG-16L, a protein essential for autophagy induction [76].

By using the I/R model of AKI, Zhou and colleagues indicated that attenuation of oxidative stress was mainly responsible for MSC-EVs-based renoprotection in AKI [77,78]. This hypothesis was based on enhanced activation of NF-E2-related factor 2/antioxidant responsive element, decreased expression of NADPH oxidase and reduced production of ROS, which were observed in the I/R-injured kidneys of MSC-EV-treated mice [77,78]. In line with these findings, $\mathrm{Gu}$ and coworkers observed preserved mitochondrial morphology in renal tubular cells of MSC-EV-treated mice [79]. They showed that miR-30 antagomirs remarkably reduced renoprotective effects of MSC-EVs, implying critical role of miR-30 in MSC-EV-based attenuation of AKI [79]. Song and colleagues further emphasized importance of MSC-derived miRNAs in renoprotection by demonstrating anti-inflammatory properties of miR-21 in alleviation of I/R-induced AKI [80]. MSC-sourced miR-21 reduced NF- $\mathrm{KB}$ activity in renal infiltrating DCs and suppressed their maturation [80]. Accordingly, administration of miR-21-containing MSC-EVs significantly attenuated capacity of renal DCs for production of inflammatory cytokines and reduced activation of Th1 and Th17 cell-driven inflammation in I/R-injured kidneys leading to the attenuation of AKI [80].

In addition to miR-21 and miR-30, members of the let-7 miR family, contained within MSC-EVs, have been shown to regulate multiple genes involved in apoptosis and proliferation of renal tubular epithelial cells, including CCNA2, CDC34, AURA/STK6, AURKB/STK12, E2F5, and CDK8 [81]. Moreover, MSC-derived let-7b was responsible for MSC-EV-induced generation of immunosuppressive M2 phenotype in renal macrophages [82]. Accordingly, significantly lower concentration of M1-derived inflammatory cytokines TNF- $\alpha$ and IL-1 $\beta$ were measured in I/R-injured kidneys of mice that received let-7b-containing MSC-EVs.

MSC-sourced miRNAs, particularly let-7c, targeted pro-fibrotic genes (collagen IV $\alpha 1$, TGF- $\beta 1$ and TGF $\beta$ R1) in inflamed kidneys, crucially contributing to the therapeutic effects of MSC-EVs in renal fibrosis and diabetic nephropathy [83-85]. In line with these findings were results obtained by Zou and colleagues who indicated that MSC-EV-dependent down-regulation of CXCL1 production was responsible for significantly decreased number of CD68+ macrophages in fibrotic kidneys 
of MSC-EVs-treated mice [84]. Since remarkably increased expression of MSC-sourced vascular endothelial growth factor (VEGF) was observed in the MSC-EV-treated kidneys, Zou and coworkers suggested that MSC-induced neo-angiogenesis was, in addition to MSC-EV-based immunosuppression, also responsible for beneficial effects of MSC-EVs in alleviation of renal fibrosis [86]. Since activation of MSCs with inflammatory cytokines (TNF- $\alpha$ and IFN- $\gamma$ ) significantly enhanced production of immunosuppressive and pro-angiogenic factors in MSCs-Exos [87], TNF- $\alpha$ and IFN- $\gamma$-priming of MSCs should be further explored as a new approach for the generation of MSC-EVs with optimal renoprotective characteristics.

\section{MSC-EV-Based Attenuation of Autoimmune and Inflammatory Eye Disease}

A large number of experimental and clinical studies demonstrated beneficial effects of MSC-Exos in the suppression of autoimmune and chronic inflammatory eye diseases [88-95]. Intravenous as well as periocular administration of MSC-Exos efficiently attenuated experimental autoimmune uveitis (EAU) $[89,90]$. MSC-Exos suppressed production of CCL2 and CCL21, which resulted in significantly reduced presence of Gr-1-expressing granulocytes, CD68-expressing macrophages and CD4+T cells in injured retinas [89]. While massive infiltration of inflammatory cells resulted in severe disruption of the retinal photoreceptor layers in vehicle-treated EAU mice, only little structural damage of retinal cells and few inflammatory infiltrates were observed in the eyes of MSC-Exo-treated EAU mice [90]. In addition to their effect on chemokine production, MSC-Exos inhibited antigen-presenting function of retinal-infiltrating DCs, as well. MSC-Exos significantly reduced expression of costimulatory molecules (CD40, CD80 and CD86) and MHC class II proteins on DCs, attenuating their capacity for activation of naive CD4+ T cells [90]. The transcript levels of DC-derived Th1 and Th17-related cytokines (IL-1 $\beta$, IL-6 and IL-12) were significantly lower in MSC-Exos-treated animals [90]. Accordingly, remarkably reduced number of IFN- $\gamma$-producing Th1 and IL-17-producing Th17 cells, that play crucially important pathogenic role in progression of EAU, were noticed in the eyes of MSC-Exo-treated EAU mice, implying that therapeutic effects of MSC-Exos in alleviation of EAU were relied on suppression of Th1 and Th17 cell-driven inflammation [90].

Th17 cells are the main inflammatory, effector cells in dry eye disease (DED), chronic inflammatory disease of the tears and ocular surface that is manifested by symptoms of discomfort, visual disturbance, and tear film instability [91]. MSC-Exos contain a growth related oncogene (GRO), which suppresses production of Th17-inducing cytokines (IL-1 $\beta$, IL-6 and IL-23) in DCs and prevent Th17 cell-driven inflammation [91,92]. In addition to GRO, MSC-sourced Indoleamine 2-3 dioxygenase (IDO) was responsible for MSC-Exo-based suppression of DC-dependent generation of Th17 cells $[93,94]$. Exos obtained from IDO-overexpressing MSCs down-regulated expression of co-stimulatory molecules and suppressed production of Th17-inducing cytokines in DCs, attenuating their capacity for activation of naïve T cells and generation of inflammatory Th17 cells $[93,94]$. Additionally, MSC-derived IDO acts as a critical molecular switch that maintains immunosuppressive phenotype of FoxP3-exspressing Tregs in inflamed tissues and prevents their re-programming into inflammatory Th17 cells [14]. Furthermore, MSC-derived IDO promotes expansion of TGF $\beta$ and IL-10-producing-immunosuppressive Tregs, contributing to the creation of immunosuppressive microenvironment in the inflamed eyes [88]. Accordingly, IDO-dependent regulation of Th17:T regulatory cells (Tregs) ratio, is also responsible for MSC-Exo-based suppression of Th17 cell driven inflammation in the eyes [88]. In line with these findings, we recently designed an ophthalmic solution (Exo-d-MAPPS), which activity was based on therapeutic effects of GRO and IDO-containing MSC-Exos [94]. Exo-d-MAPPS treatment significantly attenuated production of inflammatory cytokines in T cells and managed to alleviate dryness, grittiness, scratchiness, irritation, burning and eye fatigue in DED patients [94].

In addition to their anti-inflammatory effects, MSC-Exos promoted repair and regeneration of injured neurons in the eye [88]. Exos, obtained from bone marrow-derived MSCs, increased survival and neuritogenesis of retinal ganglion cells (RGCs) [95]. By using nerve crush model, Mead and Tomarev showed that intravitreal administration of MSC-Exos significantly reduced loss 
of RGCs and improved their function [95]. Therapeutic effects of MSC-Exos were relied on the delivery of miR-17-92, miR21 and miR-146 into the injured RGCs. MSC-sourced miR-17-92 and miR21 down-regulated expression of PTEN (an important suppressor of RGC axonal growth), while MSC-derived miR-146a reduced expression of epidermal growth factor receptor (involved in inhibition of axon regeneration) [95]. Importantly, beneficial effects of MSC-Exos in protection, repair and regeneration of RGCs were observed only in animals that received MSC-Exos and were not noticed after injection of fibroblasts-derived Exos [95], implying specific therapeutic potential of MSCs-Exos in regeneration of injured RGCs. Since gradual loss of RGCs is the hallmark of glaucoma, MSC-Exos represent potentially new therapeutic agents for glaucoma treatment, which efficacy should be explored in up-coming clinical trials.

\section{Delivery of MSC-Sourced mRNAs into the Injured Cardiomyocytes Was Mainly Responsible for MSC-EVs-Based Cardioprotection}

Several lines of evidence demonstrated that injection of MSC-EVs efficiently protected cardiomyocytes from ischemic injury $[96,97]$. By using animal model of $\mathrm{I} / \mathrm{R}$-induced myocardial injury, Lai and colleagues showed that Exos, isolated form human embryonic stem cells derived MSCs, significantly reduced infarct size and remarkably improved cardiac function in experimental animals [96]. MSC-Exos attenuated oxidative stress in I/R-injured hearts, as evidenced by greatly increased tissue levels of ATP and nicotine adenine dinucleotide and significantly decreased levels of reactive oxygen species [97]. MSC-Exos contain Parkinson protein 7/DJ-1 (DJ-1), which binds to the PARKIN protein in oxidative stress conditions, protecting the mitochondria from oxidative stress [98,99]. SinceDJ-1 protects murine heart from oxidative damage [100], MSC-sourced DJ-1 may be responsible for MSC-Exo-based modulation of oxidative balance in ischemic hearts [96]. Accordingly, Exos obtained from DJ-1-overexpressing MSCs should be explored in up-coming preclinical studies as new agents that could promote cardiac regeneration after ischemic injury. Cardioprotective effects of MSC-Exos were also relied on increased phosphorylation and activation of kinases that prevented apoptosis of injured cardiomyocytes (Akt and Glycogen synthase kinase 3 (GSK3)) and on suppression of c-Jun-N-terminal kinase, which promoted apoptosis in ischemic hearts [96].

Results obtained by $\mathrm{Yu}$ and coworkers supported the hypothesis that Akt kinase was the main intracellular target for MSC-EV-based cardioprotection [97]. They showed that Exos, obtained from Gata-4-overexpressing bone marrow derived MSCs, significantly reduced the size of ischemic lesion and restored cardiac function in the rat model of acute myocardial infarction (AMI) by activating Akt-dependent signaling pathway in injured cardiomyocytes [97]. Yu and colleagues revealed that among several MSC-Exo-containing miRNAs that regulate survival and proliferation of cardiomyocytes, miR-19a was mainly responsible for MSC-Exos-induced anti-apoptotic effects in ischemic hearts. MSC-sourced miR-19a down-regulated activation of PTEN and promoted phosphorylation and activation of Akt resulting in the up-regulation of anti-apoptotic Bcl-2 protein, resulting in reduced apoptotic loss of cardiomyocytes [97]. In line with these findings are results obtained by Wang and colleagues who demonstrated that Exos, obtained from endometrium-derived MSCs, significantly improved recovery of cardiac function after AMI by promoting Akt-dependent up-regulation of Bcl-2 activity in injured cardiomyocytes [101]. Wang et al. suggested that MSC-derived miR-21 was mainly responsible for cardioprotective effects of MSC-EVs. They demonstrated that, in addition to anti-apoptotic effects, miR21-containing MSC-Exos induced enhanced expression of vascular endothelial growth factor (VEGF) and promoted neovascularization in ischemic hearts, significantly improving cardiac function after AMI [101].

A crucially important role of MSC-sourced miRNAs for MSC-EV-based cardioprotection was confirmed by Feng and colleagues [102]. They suggested that MSC-Exo-mediated delivery of miR-22 in ischemic cardiomyocytes was mainly responsible for improved cardiac function that was noticed in MSC-Exo-treated mice with AMI [81]. Significantly reduced infarct size and cardiac fibrosis was 
a consequence of miR-22-dependent down-regulation of methyl-CpG-binding protein 2, epigenetic regulator, which was up-regulated in ischemic hearts [102].

It should be emphasized that, in addition to their anti-apoptotic effects, MSC-EVs also suppressed the influx of circulating leucocytes in injured hearts, contributing to the attenuation of on-going inflammation [97]. Significantly reduced release of alarmins and DAMPs from MSC-EV-treated cardiomyocytes resulted in decreased secretion of leucocyte-attracting chemokines by resident macrophages. Accordingly, after reperfusion, a significantly lower number of neutrophils, monocytes and lymphocytes infiltrated myocardium of MSC-Exo-treated animals, indicating that MSC-Exos-based suppression of inflammatory response also contributed to the enhanced repair and regeneration of injured cardiomyocytes [97].

\section{Conclusions and Future Directions}

MSC-EVs represent new, cell-free agents that could be used for efficient attenuation of organ-specific and systemic inflammation. Both local and systemic administration of MSC-EVs efficiently suppressed detrimental immune response in inflamed tissues and promoted survival and regeneration of injured parenchymal cells.

Through the delivery of mRNAs and miRNAs, MSC-EVs activated autophagy and/or inhibited apoptosis, necrosis and oxidative stress in injured hepatocytes, neurons, retinal cells, lung, gut and renal epithelial cells, promoting their survival and regeneration. MSC-EVs-based anti-inflammatory effects were relied on the delivery of immunoregulatory miRNAs and immunomodulatory proteins in inflammatory immune cells (M1 macrophages, DCs and Th1/Th17 cells), enabling their phenotypic conversion into anti-inflammatory and immunosuppressive cells (Table 1).

Table 1. Therapeutic effects of MSC-EVs in attenuation of inflammatory diseases.

\begin{tabular}{|c|c|c|c|c|c|c|}
\hline Disease Model & MSC Source & $\begin{array}{c}\text { Type of } \\
\text { MSC-EVs }\end{array}$ & Target Cell & Molecular Mechanism & Therapeutic Effect & Ref. No. \\
\hline DSS-induced colitis & $\mathrm{BM}$ & MSC-EVs & macrophage & $\begin{array}{c}\text { suppression of NF-kB, } \\
\text { iNOS-signaling } \\
\text { pathways }\end{array}$ & $\begin{array}{c}\text { generation of M2 } \\
\text { macrophages; } \\
\text { attenuation of colitis }\end{array}$ & [25-27] \\
\hline DSS-induced colitis & $\mathrm{UC}$ & MSC-Exos & macrophage & $\begin{array}{l}\text { suppression of } \\
\text { IL-7-signaling pathway }\end{array}$ & $\begin{array}{l}\text { increased secretion of } \\
\text { IL-10; } \\
\text { alleviation of colitis }\end{array}$ & [28] \\
\hline $\begin{array}{l}\text { D-GalN/LPS-induced } \\
\text { acute liver injury }\end{array}$ & MB & MSC-Exos & hepatocytes & $\begin{array}{l}\text { suppression of } \\
\text { caspase-3-driven } \\
\text { apoptosis }\end{array}$ & $\begin{array}{c}\text { reduced apoptosis of } \\
\text { hepatocytes; } \\
\text { increased survival } \\
\text { rate }\end{array}$ & [38] \\
\hline $\begin{array}{c}\text { Liver antigen } \\
\text { S100-induced } \\
\text { autoimmune } \\
\text { hepatitis }\end{array}$ & $\mathrm{BM}$ & MSC-Exos & hepatocytes & $\begin{array}{c}\text { inhibition of } \\
\text { caspase-1-dependent } \\
\text { pyroptosis }\end{array}$ & $\begin{array}{l}\text { attenuation of IL-1 } \beta \\
\text { and IL-18-driven } \\
\text { inflammation }\end{array}$ & [39] \\
\hline Hepatic I/R injury & iPSCs & MSC-Exos & hepatocytes & $\begin{array}{l}\text { increased activity of } \\
\text { SK1 }\end{array}$ & $\begin{array}{l}\text { increased } \\
\text { proliferation of } \\
\text { hepatocytes }\end{array}$ & {$[42,43]$} \\
\hline $\begin{array}{l}\text { CCl4-induced liver } \\
\text { fibrosis }\end{array}$ & UC & MSC-Exos & hepatocytes & $\begin{array}{c}\text { inhibition of } \\
\text { TGF- } \beta 1 / \text { Smad2 } \\
\text { signaling pathway }\end{array}$ & reduced fibrosis & [44] \\
\hline $\begin{array}{l}\text { CCl4-induced liver } \\
\text { fibrosis }\end{array}$ & $\mathrm{AM}$ & MSC-EVs & Kupffer cells & $\begin{array}{l}\text { suppressed production } \\
\text { of inflammatory } \\
\text { cytokines }\end{array}$ & $\begin{array}{l}\text { alleviated chronic } \\
\text { liver inflammation }\end{array}$ & [46] \\
\hline $\begin{array}{l}\text { CCl4-induced liver } \\
\text { fibrosis }\end{array}$ & $\mathrm{AT}$ & MSC-Exos & HSCs & $\begin{array}{l}\text { increased expression of } \\
\text { Beclin- } 1 \text { and suppressed } \\
\text { expression of Bcl-2 }\end{array}$ & $\begin{array}{c}\text { increased apoptosis } \\
\text { and autophagy of } \\
\text { HSCs; } \\
\text { attenuated fibrosis }\end{array}$ & [48] \\
\hline $\begin{array}{l}\text { I/R-induced lung } \\
\text { injury }\end{array}$ & $\mathrm{BM}$ & MSC-Exos & $\begin{array}{l}\text { lung } \\
\text { epithelial } \\
\text { cells }\end{array}$ & $\begin{array}{l}\text { suppression of } \\
\text { caspase-3,-8 and }-9\end{array}$ & $\begin{array}{c}\text { Inhibition of } \\
\text { apoptosis; } \\
\text { alleviation of lung } \\
\text { injury }\end{array}$ & [50] \\
\hline
\end{tabular}


Table 1. Cont

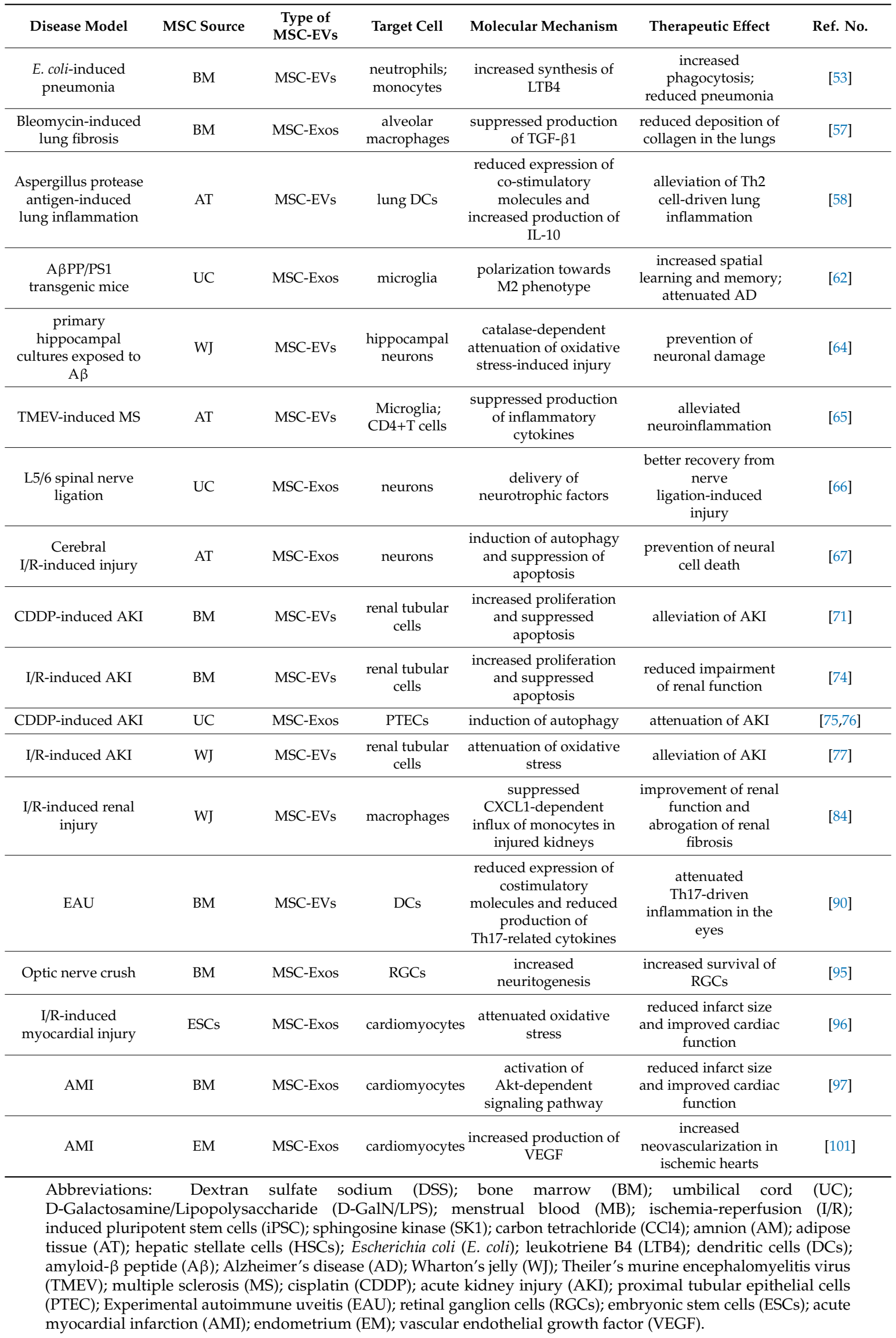


It should be noted that although experimental findings strongly suggested therapeutic potential of MSC-EVs, there is still a lot of experimental work to be done before MSC-EVs could be offered as universal human remedy for the therapy of inflammatory diseases.

MSC-EVs exhibit most of the properties of MSCs and fundamental challenges relating to MSC heterogeneity affect biological properties and therapeutic potential of MSC-EVs, as well [103]. Differences in the proliferation rate, potential for multi-lineage differentiation and immunosuppressive properties of MSCs from different sources are well-documented [104]. Furthermore, even when MSCs were obtained from the same tissue of origin, they could have prodigious donor-to-donor variation in expression of membrane markers, transcriptional and proteomic profile [104]. Aging also has a negative influence on self-renewal capacity, differentiation and immunosuppressive characteristics of MSCs, attenuating their therapeutic potential [105]. In line with these findings, several recently published studies indicated that MSC-EVs have significant tissue source and age-dependent differences in their capacity for immunosuppression and tissue regeneration [106-108]. Additionally, culture conditions in which MSCs were exposed may also influence the concentration of immunomodulatory factors within MSC-EVs. Significantly higher concentration of immunosuppressive cytokines were observed in EVs that were obtained from the MSCs, which were primed with inflammatory cytokines (TNF- $\alpha$ and IFN- $\gamma$ ) than in EVs that were derived from MSCs, which were grown under standard culture conditions [103].

Since large number of different mRNAs, miRNAs, anti-apoptotic and immunosuppressive proteins have been proposed as crucially important for beneficial effects of MSC-EVs, further experimental studies should identify the exact disease-specific MSC-sourced molecule(s) responsible for long-term protection of injured cells and/or sustained immunosuppression. Additionally, the precise dose and route of administration of MSC-EVs should be defined for each organ-specific and systemic inflammatory disease in order to prevent the development of uncontrolled immunosuppression in MSC-EVs recipients.

It should be noted that different laboratories use diverse methods to isolate and purify MSC-EVs and, accordingly, it is critical to define and standardize highly effective method for MSC-EV yields [31]. Additionally, clinical applications of MSC-EVs require their long-term use and considerable thought must be given to the preservation of their immunosuppressive potential [21]. A large number of studies demonstrated that the most convenient mode of storage for MSC-EVs remains $-80^{\circ} \mathrm{C}$ [109]. Nevertheless, due to the complex cold chain logistics, alternatives such as lyophilization and the incorporation of additives might be necessary to improve MSC-EV storage stability during transportation [21,109].

In summing up, due to their unique biological and immunosuppressive properties, MSC-EVs represents potentially new therapeutic agents in regenerative medicine. Once the critical questions around isolation, long-term preservation, donor and tissue source of MSC-EVs are answered, MSC-EVs will meet their full versatile potential as a new remedies in the therapy of inflammatory diseases.

Author Contributions: C.R.H.: manuscript writing and editing; collection of data; N.J.: manuscript writing, creation of figures; V.D.: manuscript writing and editing; collection of data; N.A.: manuscript writing; V.V.: conception and design, manuscript writing; collection of data; interpretation of data.

Funding: This work was supported by European Crohn's and Colitis Organization (ECCO) (grant “The role of galectin 3 in acute colitis"), Novartis foundation for medical-biological research (Grant No.16C197), Serbian Ministry of Science (ON175069, ON175103) and Faculty of Medical Sciences University of Kragujevac (MP01/18).

Conflicts of Interest: The authors declare no conflict of interest.

\section{References}

1. Ji, J.; Sundquist, J.; Sundquist, K. Gender-specific incidence of autoimmune diseases from national registers. J. Autoimmun. 2016, 69, 102-106. [CrossRef] [PubMed]

2. Schein, C.H. Repurposing approved drugs on the pathway to novel therapies. Med. Res. Rev. 2019. [CrossRef] [PubMed] 
3. McCaughan, G. Molecular approaches to the side effects of immunosuppressive drugs. Transplantation 2004, 78, 1114-1115. [CrossRef]

4. Regmi, S.; Pathak, S.; Kim, J.O.; Yong, C.S.; Jeong, J.H. Mesenchymal stem cell therapy for the treatment of inflammatory diseases: Challenges, opportunities, and future perspectives. Eur. J. Cell. Biol. 2019, 98, 151041. [CrossRef]

5. Volarevic, V.; Markovic, B.S.; Gazdic, M.; Volarevic, A.; Jovicic, N.; Arsenijevic, N.; Armstrong, L.; Djonov, V.; Lako, M.; Stojkovic, M. Ethical and Safety Issues of Stem Cell-Based Therapy. Int. J. Med. Sci. 2018, 15, 36-45. [CrossRef]

6. Glassberg, M.K.; Minkiewicz, J.; Toonkel, R.L.; Simonet, E.S.; Rubio, G.A.; DiFede, D.; Shafazand, S.; Khan, A.; Pujol, M.V.; LaRussa, V.F.; et al. Allogeneic Human Mesenchymal Stem Cells in Patients With Idiopathic Pulmonary Fibrosis via Intravenous Delivery (AETHER): A Phase I Safety Clinical Trial. Chest 2017, 151, 971-981. [CrossRef]

7. Duijvestein, M.; Vos, A.C.; Roelofs, H.; Wildenberg, M.E.; Wendrich, B.B.; Verspaget, H.W.; Kooy-Winkelaar, E.M.; Koning, F.; Zwaginga, J.J.; Fidder, H.H.; et al. Autologous bone marrow-derived mesenchymal stromal cell treatment for refractory luminal Crohn's disease: Results of a phase I study. Gut 2010, 59, 1662-1669. [CrossRef]

8. Dhere, T.; Copland, I.; Garcia, M.; Chiang, K.Y.; Chinnadurai, R.; Prasad, M.; Galipeau, J.; Kugathasan, S. The safety of autologous and metabolically fit bone marrow mesenchymal stromal cells in medically refractory Crohn's disease-A phase 1 trial with three doses. Aliment. Pharmacol. Ther. 2016, 44, 471-481. [CrossRef]

9. Gazdic, M.; Volarevic, V.; Arsenijevic, N.; Stojkovic, M. Mesenchymal stem cells: A friend or foe in immune-mediated diseases. Stem Cell Rev. Rep. 2015, 11, 280-287. [CrossRef]

10. Harrell, C.R.; Jankovic, M.G.; Fellabaum, C.; Volarevic, A.; Djonov, V.; Arsenijevic, A.; Volarevic, V. Molecular Mechanisms Responsible for Anti-inflammatory and Immunosuppressive Effects of Mesenchymal Stem Cell-Derived Factors. Adv. Exp. Med. Biol. 2019, 1084, 187-206.

11. Weiss, D.J.; English, K.; Krasnodembskaya, A.; Isaza-Correa, J.M.; Hawthorne, I.J.; Mahon, B.P. The Necrobiology of Mesenchymal Stromal Cells Affects Therapeutic Efficacy. Front. Immunol. 2019, 10, 1228. [CrossRef] [PubMed]

12. Otero-Ortega, L.; Gómez de Frutos, M.C.; Laso-García, F.; Rodríguez-Frutos, B.; Medina-Gutiérrez, E.; López, J.A.; Vázquez, J.; Díez-Tejedor, E.; Gutiérrez-Fernández, M. Exosomes promote restoration after an experimental animal model of intracerebral hemorrhage. J. Cereb. Blood Flow Metab. 2018, 38, 767-779. [CrossRef]

13. Matthay, M.A. Extracellular Vesicle Transfer from Mesenchymal Stromal Cells Modulates Macrophage Function in Acute Lung Injury. Basic Science and Clinical Implications. Am. J. Respir. Crit. Care Med. 2017, 196, 1234-1236. [CrossRef] [PubMed]

14. Harrell, C.R.; Fellabaum, C.; Jovicic, N.; Djonov, V.; Arsenijevic, N.; Volarevic, V. Molecular Mechanisms Responsible for Therapeutic Potential of Mesenchymal Stem Cell-Derived Secretome. Cells 2019, 8, 467. [CrossRef] [PubMed]

15. Grange, C.; Tapparo, M.; Bruno, S.; Chatterjee, D.; Quesenberry, P.J.; Tetta, C.; Camussi, G. Biodistribution of mesenchymal stem cell-derived extracellular vesicles in a model of acute kidney injury monitored by optical imaging. Int. J. Mol. Med. 2014, 33, 1055-1063. [CrossRef] [PubMed]

16. Alvarez-Erviti, L.; Seow, Y.; Yin, H.; Betts, C.; Lakhal, S.; Wood, M.J. Delivery of siRNA to the mouse brain by systemic injection of targeted exosomes. Nat. Biotechnol. 2011, 29, 341-345. [CrossRef] [PubMed]

17. Kooijmans, S.A.; Aleza, C.G.; Roffler, S.R.; van Solinge, W.W.; Vader, P.; Schiffelers, R.M. Display of GPI-anchored anti-EGFR nanobodies on extracellular vesicles promotes tumour cell targeting. J. Extracell. Vesicles 2016, 5, 31053. [CrossRef]

18. Galieva, L.R.; James, V.; Mukhamedshina, Y.O.; Rizvanov, A.A. Therapeutic Potential of Extracellular Vesicles for the Treatment of Nerve Disorders. Front. Neurosci. 2019, 13, 163. [CrossRef]

19. Matsumoto, J.; Stewart, T.; Banks, W.A.; Zhang, J. The Transport Mechanism of Extracellular Vesicles at the Blood-Brain Barrier. Curr. Pharm. Des. 2017, 23, 6206-6214. [CrossRef]

20. Morad, G.; Carman, C.V.; Hagedorn, E.J.; Perlin, J.R.; Zon, L.I.; Mustafaoglu, N.; Park, T.E.; Ingber, D.E.; Daisy, C.C.; Moses, M.A. Tumor-Derived Extracellular Vesicles Breach the Intact Blood-Brain Barrier via Transcytosis. ACS. Nano 2019. [CrossRef] 
21. Kusuma, G.D.; Barabadi, M.; Tan, J.L.; Morton, D.A.V.; Frith, J.E.; Lim, R. To Protect and to Preserve: Novel Preservation Strategies for Extracellular Vesicles. Front. Pharmacol. 2018, 9, 1199. [CrossRef] [PubMed]

22. Baumgart, D.C.; Carding, S.R. Inflammatory bowel disease: Cause and immunobiology. Lancet 2007, 369, 1627-1640. [CrossRef]

23. Lee, S.H.; Kwon, J.E.; Cho, M.L. Immunological pathogenesis of inflammatory bowel disease. Intest. Res. 2018, 16, 26-42. [CrossRef] [PubMed]

24. Wu, X.F.; Ouyang, Z.J.; Feng, L.L.; Chen, G.; Guo, W.J.; Shen, Y.; Wu, X.D.; Sun, Y.; Xu, Q. Suppression of NF- $\mathrm{B}$ signaling and NLRP3 inflammasome activation in macrophages is responsible for the amelioration of experimental murine colitis by the natural compound fraxinellone. Toxicol. Appl. Pharmacol. 2014, 281, 146-156. [CrossRef]

25. Cao, L.; Xu, H.; Wang, G.; Liu, M.; Tian, D.; Yuan, Z. Extracellular vesicles derived from bone marrow mesenchymal stem cells attenuate dextran sodium sulfate-induced ulcerative colitis by promoting M2 macrophage polarization. Int. Immunopharmacol. 2019, 72, 264-274. [CrossRef]

26. Yang, J.; Liu, X.X.; Fan, H.; Tang, Q.; Shou, Z.X.; Zuo, D.M.; Zou, Z.; Xu, M.; Chen, Q.Y.; Peng, Y.; et al. Extracellular Vesicles Derived from Bone Marrow Mesenchymal Stem Cells Protect against Experimental Colitis via Attenuating Colon Inflammation, Oxidative Stress and Apoptosis. PLoS ONE 2015, 10, e0140551. [CrossRef]

27. Wu, H.; Fan, H.; Shou, Z.; Xu, M.; Chen, Q.; Ai, C.; Dong, Y.; Liu, Y.; Nan, Z.; Wang, Y.; et al. Extracellular vesicles containing miR-146a attenuate experimental colitis by targeting TRAF6 and IRAK1. Int. Immunopharmacol. 2019, 68, 204-212. [CrossRef]

28. Mao, F.; Wu, Y.; Tang, X.; Kang, J.; Zhang, B.; Yan, Y.; Qian, H.; Zhang, X.; Xu, W. Exosomes Derived from Human Umbilical Cord Mesenchymal Stem Cells Relieve Inflammatory Bowel Disease in Mice. Biomed. Res. Int. 2017, 2017, 5356760. [CrossRef]

29. Bao, C.; Wang, B.; Yang, F.; Chen, L. Blockade of Interleukin-7 Receptor Shapes Macrophage Alternative Activation and Promotes Functional Recovery after Spinal Cord Injury. Neuroscience 2018, 371, 518-527. [CrossRef]

30. Katz, G.; Pobezinsky, L.A.; Jeurling, S.; Shinzawa, M.; Van Laethem, F.; Singer, A. T cell receptor stimulation impairs IL-7 receptor signaling by inducing expression of the microRNA miR-17 to target Janus kinase 1. Sci. Signal 2014, 7, ra83. [CrossRef]

31. Park, K.S.; Bandeira, E.; Shelke, G.V.; Lässer, C.; Lötvall, J. Enhancement of therapeutic potential of mesenchymal stem cell-derived extracellular vesicles. Stem Cell Res. Ther. 2019, 10, 288. [CrossRef] [PubMed]

32. Gazdic, M.; Simovic Markovic, B.; Vucicevic, L.; Nikolic, T.; Djonov, V.; Arsenijevic, N.; Trajkovic, V.; Lukic, M.L.; Volarevic, V. Mesenchymal stem cells protect from acute liver injury by attenuating hepatotoxicity of liver natural killer T cells in an inducible nitric oxide synthase- and indoleamine 2,3-dioxygenase-dependent manner. J. Tissue Eng. Regen. Med. 2018, 12, e1173-e1185. [CrossRef] [PubMed]

33. Gazdic, M.; Markovic, B.S.; Arsenijevic, A.; Jovicic, N.; Acovic, A.; Harrell, C.R.; Fellabaum, C.; Djonov, V.; Arsenijevic, N.; Lukic, M.L.; et al. Crosstalk between mesenchymal stem cells and T regulatory cells is crucially important for the attenuation of acute liver injury. Liver Transpl. 2018, 24, 687-702. [CrossRef]

34. Milosavljevic, N.; Gazdic, M.; Simovic Markovic, B.; Arsenijevic, A.; Nurkovic, J.; Dolicanin, Z.; Djonov, V.; Lukic, M.L.; Volarevic, V. Mesenchymal stem cells attenuate acute liver injury by altering ratio between interleukin 17 producing and regulatory natural killer T cells. Liver Transpl. 2017, 23, 1040-1050. [CrossRef]

35. Milosavljevic, N.; Gazdic, M.; Simovic Markovic, B.; Arsenijevic, A.; Nurkovic, J.; Dolicanin, Z.; Jovicic, N.; Jeftic, I.; Djonov, V.; Arsenijevic, N.; et al. Mesenchymal stem cells attenuate liver fibrosis by suppressing Th17 cells-An experimental study. Transpl. Int. 2018, 31, 102-115. [CrossRef]

36. Gazdic, M.; Arsenijevic, A.; Markovic, B.S.; Volarevic, A.; Dimova, I.; Djonov, V.; Arsenijevic, N.; Stojkovic, M.; Volarevic, V. Mesenchymal Stem Cell-Dependent Modulation of Liver Diseases. Int. J. Biol. Sci. 2017, 13, 1109-1117. [CrossRef]

37. Volarevic, V.; Gazdic, M.; Simovic Markovic, B.; Jovicic, N.; Djonov, V.; Arsenijevic, N. Mesenchymal stem cell-derived factors: Immuno-modulatory effects and therapeutic potential. Biofactors 2017, 43, 633-644. [CrossRef]

38. Chen, L.; Xiang, B.; Wang, X.; Xiang, C. Exosomes derived from human menstrual blood-derived stem cells alleviate fulminant hepatic failure. Stem Cell Res. Ther. 2017, 8, 9. [CrossRef] 
39. Chen, L.; Lu, F.B.; Chen, D.Z.; Wu, J.L.; Hu, E.D.; Xu, L.M.; Zheng, M.H.; Li, H.; Huang, Y.; Jin, X.Y.; et al. BMSCs-derived miR-223-containing exosomes contribute to liver protection in experimental autoimmune hepatitis. Mol. Immunol. 2018, 93, 38-46. [CrossRef]

40. Wu, J.; Lin, S.; Wan, B.; Velani, B.; Zhu, Y. Pyroptosis in Liver Disease: New Insights into Disease Mechanisms. Aging Dis. 2019, 10, 1094-1108. [CrossRef]

41. Yan, Y.; Jiang, W.; Tan, Y.; Zou, S.; Zhang, H.; Mao, F.; Gong, A.; Qian, H.; Xu, W. hucMSC Exosome-Derived GPX1 Is Required for the Recovery of Hepatic Oxidant Injury. Mol. Ther. 2017, 25, 465-479. [CrossRef]

42. Du, Y.; Li, D.; Han, C.; Wu, H.; Xu, L.; Zhang, M.; Zhang, J.; Chen, X. Exosomes from Human-Induced Pluripotent Stem Cell-Derived Mesenchymal Stromal Cells (hiPSC-MSCs) Protect Liver against Hepatic Ischemia/ Reperfusion Injury via Activating Sphingosine Kinase and Sphingosine-1-Phosphate Signaling Pathway. Cell. Physiol. Biochem. 2017, 43, 611-625. [CrossRef]

43. Nojima, H.; Freeman, C.M.; Schuster, R.M.; Japtok, L.; Kleuser, B.; Edwards, M.J.; Gulbins, E.; Lentsch, A.B. Hepatocyte exosomes mediate liver repair and regeneration via sphingosine-1-phosphate. J. Hepatol. 2016, 64, 60-68. [CrossRef]

44. Li, T.; Yan, Y.; Wang, B.; Qian, H.; Zhang, X.; Shen, L.; Wang, M.; Zhou, Y.; Zhu, W.; Li, W.; et al. Exosomes derived from human umbilical cord mesenchymal stem cells alleviate liver fibrosis. Stem Cells Dev. 2013, 22, 845-854. [CrossRef]

45. Kaimori, A.; Potter, J.; Kaimori, J.Y.; Wang, C.; Mezey, E.; Koteish, A. Transforming growth factor-beta1 induces an epithelial-to-mesenchymal transition state in mouse hepatocytes in vitro. J. Biol. Chem. 2007, 282, 22089-22101. [CrossRef]

46. Ohara, M.; Ohnishi, S.; Hosono, H.; Yamamoto, K.; Yuyama, K.; Nakamura, H.; Fu, Q.; Maehara, O.; Suda, G.; Sakamoto, N. Extracellular Vesicles from Amnion-Derived Mesenchymal Stem Cells Ameliorate Hepatic Inflammation and Fibrosis in Rats. Stem Cells Int. 2018, 2018, 3212643. [CrossRef]

47. Vannella, K.M.; Wynn, T.A. Mechanisms of Organ Injury and Repair by Macrophages. Annu. Rev. Physiol. 2017, 79, 593-617. [CrossRef]

48. Qu, Y.; Zhang, Q.; Cai, X.; Li, F.; Ma, Z.; Xu, M.; Lu, L. Exosomes derived from miR-181-5p-modified adipose-derived mesenchymal stem cells prevent liver fibrosis via autophagy activation. J. Cell. Mol. Med. 2017, 21, 2491-2502. [CrossRef]

49. Kim, S.Y.; Joglekar, M.V.; Hardikar, A.A.; Phan, T.H.; Khanal, D.; Tharkar, P.; Limantoro, C.; Johnson, J.; Kalionis, B.; Chrzanowski, W. Placenta Stem/Stromal Cell-Derived Extracellular Vesicles for Potential Use in Lung Repair. Proteomics 2019, 19, e1800166. [CrossRef]

50. Li, J.W.; Wei, L.; Han, Z.; Chen, Z. Mesenchymal stromal cells-derived exosomes alleviate ischemia/reperfusion injury in mouse lung by transporting anti-apoptotic miR-21-5p. Eur. J. Pharmacol. 2019, 852, 68-76. [CrossRef]

51. Bari, E.; Ferrarotti, I.; Di Silvestre, D.; Grisoli, P.; Barzon, V.; Balderacchi, A.; Torre, M.L.; Rossi, R.; Mauri, P.; Corsico, A.G.; et al. Adipose Mesenchymal Extracellular Vesicles as Alpha-1-Antitrypsin Physiological Delivery Systems for Lung Regeneration. Cells 2019, 8, 965. [CrossRef]

52. Fregonese, L.; Stolk, J. Hereditary alpha-1-antitrypsin deficiency and its clinical consequences. Orphanet J. Rare Dis. 2008, 3, 16. [CrossRef]

53. Hao, Q.; Gudapati, V.; Monsel, A.; Park, J.H.; Hu, S.; Kato, H.; Lee, J.H.; Zhou, L.; He, H.; Lee, J.W. Mesenchymal Stem Cell-Derived Extracellular Vesicles Decrease Lung Injury in Mice. J. Immunol. 2019, 203, 1961-1972. [CrossRef]

54. Mancuso, P.; Lewis, C.; Serezani, C.H.; Goel, D.; Peters-Golden, M. Intrapulmonary administration of leukotriene B4 enhances pulmonary host defense against pneumococcal pneumonia. Infect. Immun. 2010, 78, 2264-2271. [CrossRef]

55. Grabiec, A.M.; Hussell, T. The role of airway macrophages in apoptotic cell clearance following acute and chronic lung inflammation. Semin. Immunopathol. 2016, 38, 409-423. [CrossRef]

56. Huang, R.; Qin, C.; Wang, J.; Hu, Y.; Zheng, G.; Qiu, G.; Ge, M.; Tao, H.; Shu, Q.; Xu, J. Differential effects of extracellular vesicles from aging and young mesenchymal stem cells in acute lung injury. Aging 2019, 11, 7996-8014. [CrossRef]

57. Mansouri, N.; Willis, G.R.; Fernandez-Gonzalez, A.; Reis, M.; Nassiri, S.; Mitsialis, A.; Kourembanas, S. Mesenchymal stromal cell exosomes prevent and revert experimental pulmonary fibrosis through modulation of monocyte phenotypes. JCI Insight 2019, 4, 128060. [CrossRef] 
58. Cho, K.S.; Kang, S.A.; Kim, S.D.; Mun, S.J.; Yu, H.S.; Roh, H.J. Dendritic cells and M2 macrophage play an important role in suppression of Th2-mediated inflammation by adipose stem cells-derived extracellular vesicles. Stem Cell Res. 2019, 39, 101500. [CrossRef]

59. Guilliams, M.; Lambrecht, B.N.; Hammad, H. Division of labor between lung dendritic cells and macrophages in the defense against pulmonary infections. Mucosal. Immunol. 2013, 6, 464-473. [CrossRef]

60. Lambrecht, B.N.; Prins, J.B.; Hoogsteden, H.C. Lung dendritic cells and host immunity to infection. Eur. Respir. J. 2001, 18, 692-704.

61. Subhramanyam, C.S.; Wang, C.; Hu, Q.; Dheen, S.T. Microglia-mediated neuroinflammation in neurodegenerative diseases. Semin. Cell. Dev. Biol. 2019, 94, 112-120. [CrossRef] [PubMed]

62. Ding, M.; Shen, Y.; Wang, P.; Xie, Z.; Xu, S.; Zhu, Z.; Wang, Y.; Lyu, Y.; Wang, D.; Xu, L.; et al. Exosomes Isolated From Human Umbilical Cord Mesenchymal Stem Cells Alleviate Neuroinflammation and Reduce Amyloid-Beta Deposition by Modulating Microglial Activation in Alzheimer's Disease. Neurochem. Res. 2018, 43, 2165-2177. [CrossRef] [PubMed]

63. Barnett, R. Alzheimer's disease. Lancet 2019, 393, 1589. [CrossRef]

64. Bodart-Santos, V.; de Carvalho, L.R.P.; de Godoy, M.A.; Batista, A.F.; Saraiva, L.M.; Lima, L.G.; Abreu, C.A.; De Felice, F.G.; Galina, A.; Mendez-Otero, R.; et al. Extracellular vesicles derived from human Wharton's jelly mesenchymal stem cells protect hippocampal neurons from oxidative stress and synapse damage induced by amyloid- $\beta$ oligomers. Stem Cell Res. Ther. 2019, 10, 332. [CrossRef]

65. Laso-García, F.; Ramos-Cejudo, J.; Carrillo-Salinas, F.J.; Otero-Ortega, L.; Feliú, A.; Gómez-de Frutos, M.; Mecha, M.; Díez-Tejedor, E.; Guaza, C.; Gutiérrez-Fernández, M. Therapeutic potential of extracellular vesicles derived from human mesenchymal stem cells in a model of progressive multiple sclerosis. PLoS ONE 2018, 13, e0202590. [CrossRef]

66. Shiue, S.J.; Rau, R.H.; Shiue, H.S.; Hung, Y.W.; Li, Z.X.; Yang, K.D.; Cheng, J.K. Mesenchymal stem cell exosomes as a cell-free therapy for nerve injury-induced pain in rats. Pain 2019, 160, 210-223. [CrossRef]

67. Huang, X.; Ding, J.; Li, Y.; Liu, W.; Ji, J.; Wang, H.; Wang, X. Exosomes derived from PEDF modified adipose-derived mesenchymal stem cells ameliorate cerebral ischemia-reperfusion injury by regulation of autophagy and apoptosis. Exp. Cell. Res. 2018, 371, 269-277. [CrossRef]

68. Yabe, T.; Sanagi, T.; Yamada, H. The neuroprotective role of PEDF: Implication for the therapy of neurological disorders. Curr. Mol. Med. 2010, 10, 259-266. [CrossRef]

69. Tsuji, K.; Kitamura, S.; Wada, J. Secretomes from Mesenchymal Stem Cells against Acute Kidney Injury: Possible Heterogeneity. Stem Cells Int. 2018, 2018, 8693137. [CrossRef]

70. Bruno, S.; Grange, C.; Deregibus, M.C.; Calogero, R.A.; Saviozzi, S.; Collino, F.; Morando, L.; Busca, A.; Falda, M.; Bussolati, B.; et al. Mesenchymal stem cell-derived microvesicles protect against acute tubular injury. J. Am. Soc. Nephrol. 2009, 20, 1053-1067. [CrossRef]

71. Bruno, S.; Tapparo, M.; Collino, F.; Chiabotto, G.; Deregibus, M.C.; Soares Lindoso, R.; Neri, F.; Kholia, S.; Giunti, S.; Wen, S.; et al. Renal Regenerative Potential of Different Extracellular Vesicle Populations Derived from Bone Marrow Mesenchymal Stromal Cells. Tissue Eng. Part A 2017, 23, 1262-1273. [CrossRef]

72. Bruno, S.; Grange, C.; Collino, F.; Deregibus, M.C.; Cantaluppi, V.; Biancone, L.; Tetta, C.; Camussi, G. Microvesicles derived from mesenchymal stem cells enhance survival in a lethal model of acute kidney injury. PLoS ONE 2012, 7, e33115. [CrossRef]

73. Ju, G.Q.; Cheng, J.; Zhong, L.; Wu, S.; Zou, X.Y.; Zhang, G.Y.; Gu, D.; Miao, S.; Zhu, Y.J.; Sun, J.; et al. Microvesicles derived from human umbilical cord mesenchymal stem cells facilitate tubular epithelial cell dedifferentiation and growth via hepatocyte growth factor induction. PLOS ONE 2015, 10, e0121534. [CrossRef]

74. Gatti, S.; Bruno, S.; Deregibus, M.C.; Sordi, A.; Cantaluppi, V.; Tetta, C.; Camussi, G. Microvesicles derived from human adult mesenchymal stem cells protect against ischaemia-reperfusion-induced acute and chronic kidney injury. Nephrol. Dial. Transplant. 2011, 26, 1474-1483. [CrossRef]

75. Wang, B.; Jia, H.; Zhang, B.; Wang, J.; Ji, C.; Zhu, X.; Yan, Y.; Yin, L.; Yu, J.; Qian, H.; et al. Pre-incubation with hucMSC-exosomes prevents cisplatin-induced nephrotoxicity by activating autophagy. Stem Cell Res. Ther. 2017, 8, 75. [CrossRef]

76. Jia, H.; Liu, W.; Zhang, B.; Wang, J.; Wu, P.; Tandra, N.; Liang, Z.; Ji, C.; Yin, L.; Hu, X.; et al. HucMSC exosomes-delivered 14-3-3 $\zeta$ enhanced autophagy via modulation of ATG16L in preventing cisplatin-induced acute kidney injury. Am. J. Transl. Res. 2018, 10, 101-113. 
77. Zhang, G.; Zou, X.; Miao, S.; Chen, J.; Du, T.; Zhong, L.; Ju, G.; Liu, G.; Zhu, Y. The anti-oxidative role of micro-vesicles derived from human Wharton-Jelly mesenchymal stromal cells through NOX2/gp91(phox) suppression in alleviating renal ischemia-reperfusion injury in rats. PLoS ONE 2014, 9, e92129. [CrossRef]

78. Zhang, G.; Zou, X.; Huang, Y.; Wang, F.; Miao, S.; Liu, G.; Chen, M.; Zhu, Y. Mesenchymal Stromal Cell-Derived Extracellular Vesicles Protect Against Acute Kidney Injury Through Anti-Oxidation by Enhancing Nrf2/ARE Activation in Rats. Kidney Blood Press. Res. 2016, 41, 119-128. [CrossRef]

79. Gu, D.; Zou, X.; Ju, G.; Zhang, G.; Bao, E.; Zhu, Y. Mesenchymal Stromal Cells Derived Extracellular Vesicles Ameliorate Acute Renal Ischemia Reperfusion Injury by Inhibition of Mitochondrial Fission through miR-30. Stem Cells Int. 2016, 2016, 2093940. [CrossRef]

80. Song, N.; Zhang, T.; Xu, X.; Lu, Z.; Yu, X.; Fang, Y.; Hu, J.; Jia, P.; Teng, J.; Ding, X. miR-21 Protects Against Ischemia/Reperfusion-Induced Acute Kidney Injury by Preventing Epithelial Cell Apoptosis and Inhibiting Dendritic Cell Maturation. Front. Physiol. 2018, 9, 790. [CrossRef]

81. Johnson, C.D.; Esquela-Kerscher, A.; Stefani, G.; Byrom, M.; Kelnar, K.; Ovcharenko, D.; Wilson, M.; Wang, X.; Shelton, J.; Shingara, J.; et al. The let-7 microRNA represses cell proliferation pathways in human cells. Cancer Res. 2007, 67, 7713-7722. [CrossRef]

82. Ti, D.; Hao, H.; Tong, C.; Liu, J.; Dong, L.; Zheng, J.; Zhao, Y.; Liu, H.; Fu, X.; Han, W. LPS-preconditioned mesenchymal stromal cells modify macrophage polarization for resolution of chronic inflammation via exosome-shuttled let-7b. J. Transl. Med. 2015, 13, 308. [CrossRef] [PubMed]

83. Wang, B.; Yao, K.; Huuskes, B.M.; Shen, H.H.; Zhuang, J.; Godson, C.; Brennan, E.P.; Wilkinson-Berka, J.L.; Wise, A.F.; Ricardo, S.D. Mesenchymal Stem Cells Deliver Exogenous MicroRNA-let7c via Exosomes to Attenuate Renal Fibrosis. Mol. Ther. 2016, 24, 1290-1301. [CrossRef] [PubMed]

84. Zou, X.; Zhang, G.; Cheng, Z.; Yin, D.; Du, T.; Ju, G.; Miao, S.; Liu, G.; Lu, M.; Zhu, Y. Microvesicles derived from human Wharton's Jelly mesenchymal stromal cells ameliorate renal ischemia-reperfusion injury in rats by suppressing CX3CL1. Stem Cell Res. Ther. 2014, 5, 40. [CrossRef]

85. Grange, C.; Tritta, S.; Tapparo, M.; Cedrino, M.; Tetta, C.; Camussi, G.; Brizzi, M.F. Stem cell-derived extracellular vesicles inhibit and revert fibrosis progression in a mouse model of diabetic nephropathy. Sci. Rep. 2019, 9, 4468. [CrossRef]

86. Zou, X.; Gu, D.; Xing, X.; Cheng, Z.; Gong, D.; Zhang, G.; Zhu, Y. Human mesenchymal stromal cell-derived extracellular vesicles alleviate renal ischemic reperfusion injury and enhance angiogenesis in rats. Am. J. Transl. Res. 2016, 8, 4289-4299.

87. Domenis, R.; Cifù, A.; Quaglia, S.; Pistis, C.; Moretti, M.; Vicario, A.; Parodi, P.C.; Fabris, M.; Niazi, K.R.; Soon-Shiong, P.; et al. Pro inflammatory stimuli enhance the immunosuppressive functions of adipose mesenchymal stem cells-derived exosomes. Sci. Rep. 2018, 8, 13325. [CrossRef]

88. Harrell, C.R.; Simovic Markovic, B.; Fellabaum, C.; Arsenijevic, A.; Djonov, V.; Arsenijevic, N.; Volarevic, V. Therapeutic Potential of Mesenchymal Stem Cell-Derived Exosomes in the Treatment of Eye Diseases. Adv. Exp. Med. Biol. 2018, 1089, 47-57.

89. Bai, L.; Shao, H.; Wang, H.; Zhang, Z.; Su, C.; Dong, L.; Yu, B.; Chen, X.; Li, X.; Zhang, X. Effects of Mesenchymal Stem Cell-Derived Exosomes on Experimental Autoimmune Uveitis. Sci. Rep. 2017, 7, 4323. [CrossRef]

90. Shigemoto-Kuroda, T.; Oh, J.Y.; Kim, D.K.; Jeong, H.J.; Park, S.Y.; Lee, H.J.; Park, J.W.; Kim, T.W.; An, S.Y.; Prockop, D.J.; et al. MSC-derived Extracellular Vesicles Attenuate Immune Responses in Two Autoimmune Murine Models: Type 1 Diabetes and Uveoretinitis. Stem Cell Rep. 2017, 8, 1214-1225. [CrossRef]

91. Gayton, J.L. Etiology, prevalence, and treatment of dry eye disease. Clin. Ophthalmol. 2009, 3, 405-412. [CrossRef] [PubMed]

92. De Paiva, C.S.; Chotikavanich, S.; Pangelinan, S.B.; Pitcher, J.D., 3rd; Fang, B.; Zheng, X.; Ma, P.; Farley, W.J.; Siemasko, K.F.; Niederkorn, J.Y.; et al. IL-17 disrupts corneal barrier following desiccating stress. Mucosal Immunol. 2009, 2, 243-253. [CrossRef] [PubMed]

93. He, J.G.; Xie, Q.L.; Li, B.B.; Zhou, L.; Yan, D. Exosomes Derived from IDO1-Overexpressing Rat Bone Marrow Mesenchymal Stem Cells Promote Immunotolerance of Cardiac Allografts. Cell Transplant. 2018, 27, 1657-1683. [CrossRef] 
94. Harrell, C.R.; Fellabaum, C.; Simovic Markovic, B.; Arsenijevic, A.; Volarevic, V. Therapeutic Potential of "Exosomes Derived Multiple Allogeneic Proteins Paracrine Signaling: Exosomes D-Mapps" is Based on the Effects of Exosomes, Immunosuppressive and Trophic Factors. Ser. J. Exp. Clin. Res. 2019, 20, 189-197. [CrossRef]

95. Mead, B.; Tomarev, S. Bone Marrow-Derived Mesenchymal Stem Cells-Derived Exosomes Promote Survival of Retinal Ganglion Cells Through miRNA-Dependent Mechanisms. Stem Cells Transl. Med. 2017, 6, 1273-1285. [CrossRef]

96. Lai, R.C.; Arslan, F.; Lee, M.M.; Sze, N.S.; Choo, A.; Chen, T.S.; Salto-Tellez, M.; Timmer, L.; Lee, C.N.; El Oakley, R.M.; et al. Exosome secreted by MSC reduces myocardial ischemia/reperfusion injury. Stem Cell Res. 2010, 4, 214-222. [CrossRef]

97. Yu, B.; Kim, H.W.; Gong, M.; Wang, J.; Millard, R.W.; Wang, Y.; Ashraf, M.; Xu, M. Exosomes secreted from GATA-4 overexpressing mesenchymal stem cells serve as a reservoir of anti-apoptotic microRNAs for cardioprotection. Int. J. Cardiol. 2015, 182, 349-360. [CrossRef]

98. Vilaça-Faria, H.; Salgado, A.J.; Teixeira, F.G. Mesenchymal Stem Cells-derived Exosomes: A New Possible Therapeutic Strategy for Parkinson's Disease? Cells 2019, 8, 118. [CrossRef]

99. Moore, D.J.; Zhang, L.; Troncoso, J.; Lee, M.K.; Hattori, N.; Mizuno, Y.; Dawson, T.M.; Dawson, V.L. Association of DJ-1 and parkin mediated by pathogenic DJ-1 mutations and oxidative stress. Hum. Mol. Genet. 2005, 14, 71-84. [CrossRef]

100. Billia, F.; Hauck, L.; Grothe, D.; Konecny, F.; Rao, V.; Kim, R.H.; Mak, T.W. Parkinson-susceptibility gene DJ-1/PARK7 protects the murine heart from oxidative damage in vivo. Proc. Natl. Acad. Sci. USA 2013, 110, 6085-6090. [CrossRef]

101. Wang, K.; Jiang, Z.; Webster, K.A.; Chen, J.; Hu, H.; Zhou, Y.; Zhao, J.; Wang, L.; Wang, Y.; Zhong, Z.; et al. Enhanced Cardioprotection by Human Endometrium Mesenchymal Stem Cells Driven by Exosomal MicroRNA-21. Stem Cells Transl. Med. 2017, 6, 209-222. [CrossRef] [PubMed]

102. Feng, Y.; Huang, W.; Wani, M.; Yu, X.; Ashraf, M. Ischemic preconditioning potentiates the protective effect of stem cells through secretion of exosomes by targeting Mecp2 via miR-22. PLoS ONE 2014, 9, e88685. [CrossRef] [PubMed]

103. Lai, P.; Weng, J.; Guo, L.; Chen, X.; Du, X. Novel insights into MSC-EVs therapy for immune diseases. Biomark. Res. 2019, 7, 6. [CrossRef] [PubMed]

104. Wilson, A.; Hodgson-Garms, M.; Frith, J.E.; Genever, P. Multiplicity of Mesenchymal Stromal Cells: Finding the Right Route to Therapy. Front. Immunol. 2019, 10, 1112. [CrossRef]

105. Li, Y.; Wu, Q.; Wang, Y.; Li, L.; Bu, H.; Bao, J. Senescence of mesenchymal stem cells (Review). Int. J. Mol. Med. 2017, 39, 775-782. [CrossRef]

106. Fafián-Labora, J.; Lesende-Rodriguez, I.; Fernández-Pernas, P.; Sangiao-Alvarellos, S.; Monserrat, L.; Arntz, O.J.; van de Loo, F.J.; Mateos, J.; Arufe, M.C. Effect of age on pro-inflammatory miRNAs contained in mesenchymal stem cell-derived extracellular vesicles. Sci. Rep. 2017, 7, 43923. [CrossRef]

107. Bobis-Wozowicz, S.; Kmiotek, K.; Kania, K.; Karnas, E.; Labedz-Maslowska, A.; Sekula, M.; Kedracka-Krok, S.; Kolcz, J.; Boruczkowski, D.; Madeja, Z.; et al. Diverse impact of xeno-free conditions on biological and regenerative properties of hUC-MSCs and their extracellular vesicles. J. Mol. Med. 2017, 95, 205-220. [CrossRef]

108. Kordelas, L.; Schwich, E.; Dittrich, R.; Horn, P.A.; Beelen, D.W.; Börger, V.; Giebel, B.; Rebmann, V. Individual Immune-Modulatory Capabilities of MSC-Derived Extracellular Vesicle (EV) Preparations and Recipient-Dependent Responsiveness. Int. J. Mol. Sci. 2019, 20, 1642. [CrossRef]

109. Jeyaram, A.; Jay, S.M. Preservation and Storage Stability of Extracellular Vesicles for Therapeutic Applications. AAPS J. 2017, 20, 1. [CrossRef]

(C) 2019 by the authors. Licensee MDPI, Basel, Switzerland. This article is an open access article distributed under the terms and conditions of the Creative Commons Attribution (CC BY) license (http://creativecommons.org/licenses/by/4.0/). 